$\underline{\text { Preprint typeset in JHEP style - PAPER VERSION }}$

ITP-UU-08/70

NIKHEF-2008-33

ITFA-2008-44

\title{
Path integral approach to eikonal and next-to-eikonal exponentiation
}

\author{
Eric Laenen \\ ITFA, University of Amsterdam, Valckenierstraat 65, 1018 XE Amsterdam, \\ ITF, Utrecht University, Leuvenlaan 4, 3584 CE Utrecht \\ Nikhef Theory Group, Kruislaan 409, 1098 SJ Amsterdam, The Netherlands \\ E-mail: Eric.Laenen@nikhef.nl
}

\author{
Gerben Stavenga \\ Institute for Theoretical Physics, Utrecht University, Leuvenlaan 4, 3584 CE Utrecht, \\ The Netherlands \\ E-mail: stavenga@phys.uu.nl
}

\author{
Chris D. White \\ Nikhef Theory Group, Kruislaan 409, 1098 SJ Amsterdam, The Netherlands \\ E-mail: cwhite@nikhef.nl
}

\begin{abstract}
We approach the issue of exponentiation of soft gauge boson corrections to scattering amplitudes from a path integral point of view. We show that if one represents the amplitude as a first quantized path integral in a mixed coordinate-momentum space representation, a charged particle interacting with a soft gauge field is represented as a Wilson line for a semi-infinite line segment, together with calculable fluctuations. Combining such line segments, we show that exponentiation in an abelian field theory follows immediately from standard path-integral combinatorics. In the non-abelian case, we consider color singlet hard interactions with two outgoing external lines, and obtain a new viewpoint for exponentiation in terms of "webs", with a closed form solution for their corresponding color factors. We investigate and clarify the structure of next-to-eikonal corrections.
\end{abstract}




\section{Contents}

1. Introduction 1

1.1 Propagators as first quantized path integrals 4

1.1.1 Free scalar particle 4

1.2 Scalar particle in an abelian background gauge field 6

$\begin{array}{lll}1.3 & \text { Spinor particle } & 7\end{array}$

2. Soft emissions in scattering processes $\quad 8$

2.1 Eikonal exponentiation 11

2.2 Next-to-eikonal exponentiation 12

2.3 Exponentiation for spinor particles 13

2.4 Low's theorem 14

3. Non-abelian gauge theory $\quad 18$

3.1 Non-abelian exponentiation at NE order $\quad 27$

$\begin{array}{lll}3.2 & \text { Internal emissions of non-abelian gauge fields } 30\end{array}$

$\begin{array}{ll}\text { 4. Discussion } & 31\end{array}$

A. Exponentiation of disconnected diagrams 33

B. Next-to-eikonal Feynman rules $\quad 34$

C. Matrix elements with internal emissions 40

\section{Introduction}

Higher order corrections arising from soft gauge bosons in perturbative gauge theory, be they real or virtual, have been the subject of many investigations. Such radiation generically leads to series of perturbative contributions to differential cross-sections of the form $\alpha^{n} \log ^{m}(\xi) / \xi$, where $\alpha$ is the coupling constant of the gauge theory, and $\xi$ is related to the energy carried away by the soft particles. In the soft limit $\xi \rightarrow 0$ (in which the eikonal approximation may be taken), it becomes necessary to resum these terms to all orders in perturbation theory, as has been achieved by a variety of methods $[1,2,3,4,5,6,7,8]$. Central to resummation is the exponentiation of eikonalized soft gauge boson corrections and it has been shown for both abelian and non-abelian gauge theory that this indeed occurs $[9,10,11,12]$.

To form an exponential series for a cross-section, both the matrix element and the phase space must exhibit an appropriate factorized structure. The statement for the matrix 

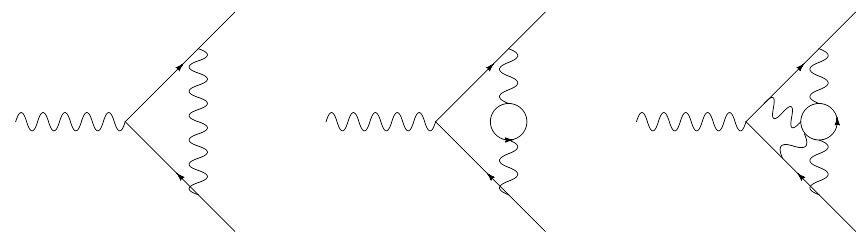

Figure 1: Examples of connected diagrams $G_{c}$ of soft emissions between hard outgoing particle legs in abelian perturbation theory.
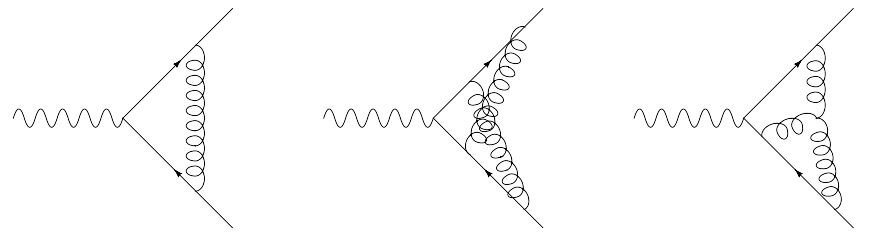

Figure 2: Examples of webs of soft emissions between external lines in non-abelian perturbation theory.

elements rests upon a thorough analysis of the general structure of higher order diagrams. The result for an amplitude $\mathcal{A}$ in abelian gauge theory is simply expressed in the eikonal approximation as

$$
\mathcal{A}=\mathcal{A}_{0} \exp \left[\sum G_{c}\right]
$$

where $\mathcal{A}$ is the amplitude without soft radiation containing a number of hard outgoing external lines, and the sum in the exponent is over connected diagrams $G_{c}$ between the external lines, with the Born contribution $\mathcal{A}_{0}$ factored out. Examples are shown for the case of hard production of a particle-antiparticle pair in figure 1. The non-abelian case is complicated by the nontrivial color structure of the Feynman diagrams at successive orders in perturbation theory. Nevertheless, in the case of two external lines ${ }^{1}$, exponentiation still holds provided one generalizes Eq. (1.1) to

$$
\mathcal{A}=\mathcal{A}_{0} \exp \left[\sum \bar{C}_{W} W\right]
$$

Here $W$ are so-called webs, and are diagrams which are two-eikonal irreducible. That is, one cannot partition a web into webs of lower order by cutting both external lines exactly once $[10,11,12]^{2}$. Examples are shown in figure 2. Each web has an associated color factor $\bar{C}_{W}$ which is not the same as the normal color factor $C_{W}$ associated with the web graph. The color factors $\bar{C}_{W}$ are given in terms of $C_{W}$ by an iterative relation to all orders in perturbation theory.

The nature of Eq.( 1.1) in terms of disconnected diagrams is reminiscent of another well-known property of quantum field theory, namely the exponentiation of disconnected Feynman diagrams in terms of connected ones. This latter property most naturally emerges

\footnotetext{
${ }^{1}$ In the case of more than two external lines, the structure is more complicated.

${ }^{2}$ See [13] for a pedagogical exposition.
} 
using path integral methods (see appendix A for a brief proof), and thus the suggestion arises of whether it is possible to relate the exponentiation of soft radiative corrections to the gauge theory path integral. The aim of this paper is to show that this is indeed the case, and the result is important for a number of reasons. Firstly, it provides a new perspective on exponentiation. Secondly, it allows one to straightforwardly explore which properties of exponentiation survive at next-to-eikonal limit and beyond (i.e. corresponding to subleading terms in $\xi$ above $)^{3}$. Although at next-to-eikonal order fermion emissions also contribute (leading to flavor-changing effects), we restrict ourselves here to gluon emissions.

The essential idea of our approach is as follows. One first relates the field theory path integral for a particle interacting with a gauge field to a first-quantized path integral with respect to the particle. That is, the external lines become worldlines of particles in quantum mechanics (rather than quantum field theory). Here we utilize the techniques of $[14,15,16]$ which have originally been applied in a different context (that of constructing string theoretical analogues of fixed order field theory amplitudes [17, 18]). A first-quantized approach to Sudakov resummation has also appeared in [19]. We will see explicitly that in this representation the eikonal limit corresponds to the radiating particles moving classically, and next-to-eikonal terms originate from fluctuations around the classical path. The soft radiation emission vertices can then be interpreted as interactions of the gauge field with a source, such that individual emission vertices form disconnected diagrams. Then exponentiation of eikonal corrections follows naturally from usual combinatoric properties of the path integral.

In the non-abelian case, exponentiation is complicated by the fact that vertices for the emissions of gluons do not commute. However, one can rephrase the problem using the replica trick of statistical physics (see e.g. [20]), such that a subset of diagrams arises which exponentiate. These are then precisely the webs of $[10,11]$. Furthermore, we provide an explicit closed form solution for the modified color factors, given in terms of normal (rather than modified) color weights.

Our formalism allows one to straightforwardly consider subleading effects with respect to the eikonal limit, and we classify the possible next-to-eikonal corrections. This can be divided into a subset which exponentiate (involving NE generalizations of the webs discussed above), and a set of remainder terms which do not formally exponentiate, but have an iterative structure in that each order of the perturbation expansion is sufficient to generate the next order.

Earlier attempts to include certain sub-eikonal effects were done in practical implementations of Sudakov resummation, mostly in view of gauging the theoretical uncertainty of the resummation [21]. Typically, this involved including subleading terms in the collinear evolution kernel in the resummation, which is particularly appealing for Drell-Yan, Higgs production and related cross sections $[21,22,23,24,25,26]$. More recently a study was performed [27] based on a proposal in Ref. [28].

In the rest of this introduction we review the derivation of the path-integral representation of propagators in quantum field theory, for both scalar and spinor particles and in the

\footnotetext{
${ }^{3}$ We only consider matrix elements in this paper. Exponentiation in differential cross-sections also depends upon factorization of the multiple particle phase space, and is deferred for further study.
} 


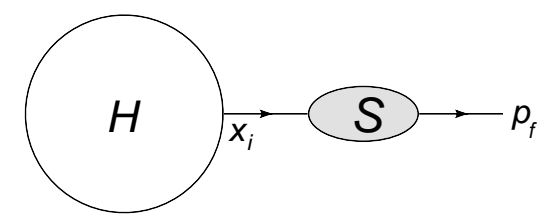

Figure 3: Anatomy of an external line, considered throughout this paper as emerging from a hard interaction at 4 -position $x_{i}$ at time $t=0$, and having momentum $p_{f}$ at final time $T$. The propagator for the external line contains the effects of soft radiation.

presence and absence of an abelian gauge field. These results will be used in section 2 to demonstrate the exponentiation of soft radiative corrections in the presence of an abelian gauge field. We also consider generalization of the results to beyond the eikonal limit, and classify the resulting corrections into a subset which exponentiate and a remainder term which mixes with these at next-to-eikonal order. In section 3 we consider the extension to non-abelian gauge fields, recovering the properties of webs and again examining corrections to the eikonal limit. We conclude in section 4, and some technical details are presented in the appendices.

\subsection{Propagators as first quantized path integrals}

Throughout this paper we will be concerned with external lines, namely hard external particles susceptible to soft radiation emission. A given external line is created from a hard interaction process at time $t=0$ at space-time point $x_{i}$, and and has a momentum $p_{f}$ at some final time $T \rightarrow \infty$ (see figure 3). Soft radiation corrections enter the twopoint function for the emitting particle i.e. the propagator for the external line. In the following subsections, we review the representation of field theory propagators in terms of first quantized path integrals $[14,15,16]$, which will later on be used in the derivation of matrix element exponentiation at eikonal order and beyond. We begin with the simplest case, that of a free scalar particle.

\subsubsection{Free scalar particle}

The propagator for a free scalar particle between 4-positions $x$ and $y$ is the Green's function for the Klein-Gordon equation

$$
i(S-i \varepsilon) \Delta_{F}(x, y)=\delta^{(d)}(x, y), \quad S=\left(-\square_{x}+m^{2}\right),
$$

where $S, \Delta_{F}$ are Hermitian operators working on the Hilbert space $\mathcal{H}$ of square integrable functions of space-time, and we adopt the standard Feynman $i \varepsilon$ prescription. Note that we are using the metric $(-,+,+,+)$. Schematically the propagator may be written as

$$
\Delta_{F}=[i(S-i \varepsilon)]^{-1},
$$

where the inverse operator can be defined via an inverse Fourier transform from momentum space. The usual representation then reads

$$
\Delta_{F}(x, y)=-i \int \frac{d^{d} p}{(2 \pi)^{d}} \frac{e^{i p \cdot(y-x)}}{p^{2}+m^{2}-i \varepsilon} .
$$


To derive this expression using a first-quantized path integral, let us first write the inverse Klein-Gordon operator using the Schwinger representation

$$
-i(S-i \varepsilon)^{-1}=\frac{1}{2} \int_{0}^{\infty} d T e^{-i \frac{1}{2}(S-i \varepsilon) T}
$$

where the $i \varepsilon$ now ensures the convergence of the integral. The integrand contains the exponential $U(T)=e^{-i \frac{1}{2} S T}$, which is a unitary operator acting on the Hilbert space $\mathcal{H}$ and satisfying the Schrödinger equation

$$
i \frac{d}{d T} U(T)=\hat{H} U(T), \quad U(0)=I, \quad \hat{H}=\frac{1}{2} S .
$$

We can therefore interpret $\hat{H}$ as the Hamiltonian operator of a quantum system, with internal time coordinate $T$. Given we are considering external lines as shown in figure 3, we must calculate the expectation value of the evolution operator $U$ between a state of definite position (at time $t=0$ ) and definite momentum (at time $t=T$ ). To do this we introduce states $|x\rangle$ and $|p\rangle$ in the Hilbert space $\mathcal{H}$, and write the Hamiltonian as

$$
\hat{H}(\hat{x}, \hat{p})=\sum_{n=0}^{\infty} \hat{p}_{\mu_{1}} \ldots \hat{p}_{\mu_{n}} H_{\nu_{1} \ldots \nu_{n}}^{\mu_{1} \ldots \mu_{n}} \hat{x}^{\nu_{1} \ldots \nu_{n}}
$$

where $\hat{x}$ and $\hat{p}$ are the position and momentum operators whose continuous eigenstates are $|x\rangle$ and $|p\rangle$ respectively. Note we have expressed $\hat{H}$ in Weyl ordered form, with all momentum operators to the left of position operators. One then finds, for small time separations $\Delta t$

$$
\left\langle p\left|e^{-i H \Delta t}\right| x\right\rangle=e^{-i H(p, x) \Delta t+O\left[(\Delta t)^{2}\right]}\langle p \mid x\rangle,
$$

where $H(p, x)$ is the c-number obtained by replacing the operators on the right-hand-side of Eq. (1.8) with their corresponding variables. Slicing the time variable into $N$ steps of duration $\Delta t$ and inserting a complete set of both position and momentum states at each step, Eq. (1.9) becomes

$$
\int d x_{1} \ldots d x_{N} \int d p_{0} \ldots d p_{N-1} \exp \left[-i \sum_{k=0}^{N-1} H\left(p_{k}, x_{k}\right) \Delta t\right] \prod_{k=0}^{N}\left\langle p_{k} \mid x_{k}\right\rangle \prod_{k=0}^{N-1}\left\langle x_{k+1} \mid p_{k}\right\rangle .
$$

Using the normalization of the basis states

$$
\langle x \mid p\rangle=\frac{e^{i x p}}{(2 \pi)^{d}}
$$

where $d$ is the number of space-time dimensions, the continuum limit of Eq.(1.10) is

$$
\left\langle p_{f}|U(T)| x_{i}\right\rangle=\int_{x(0)=x_{i}}^{p(T)=p_{f}} \mathcal{D} p \mathcal{D} x \exp \left[-i p(T) x(T)+i \int_{0}^{T} d t(p \dot{x}-H(p, x))\right] .
$$

We have absorbed factors of $2 \pi$ into the measure, and made the boundary conditions explicit. This is the well-known path-integral result for the evolution operator sandwiched 
between initial and final position states, with an additional term in the exponent involving $p(T) x(T)$ arising from considering a final state of given momentum, rather than position.

For the present case of a free massive scalar, the Hamiltonian function is given by

$$
H(p)=\frac{1}{2}\left(p^{2}+m^{2}\right) .
$$

We can perform the path integrations over $p(t)$ and $x(t)$ by expanding around the classical solution of the equations of motion, given by

$$
p(t)=p_{f}+p^{\prime}(t), \quad x(t)=x_{i}+p_{f} t+x^{\prime}(t) .
$$

The boundary conditions imply $p^{\prime}(T)=0$ and $x^{\prime}(0)=0$, and without confusion we can drop the primed notation from now on. Substituting Eq. (1.14) into Eq. (1.12) gives

$$
\left\langle p_{f}|U(T)| x_{i}\right\rangle=e^{-i p_{f} x_{i}-i \frac{1}{2}\left(p_{f}^{2}+m^{2}\right) T} \int_{x(0)=0}^{p(T)=0} \mathcal{D} p \mathcal{D} x e^{i \int_{0}^{T} d t\left(p \dot{x}-\frac{1}{2} p^{2}\right)} .
$$

One can now perform the path integral as the continuum limit of a product of Gaussian integrals in the intermediate position and momentum variables. The measure is such that this gives unity, and one therefore finds

$$
\left\langle p_{f}|U(T)| x_{i}\right\rangle=e^{-i p_{f} x_{i}-\frac{1}{2} i\left(p_{f}^{2}+m^{2}\right) T} .
$$

The momentum space propagator $\tilde{\Delta}_{F}$ is found by substituting Eq. (1.16) into Eq. (1.6), and one finds

$$
\tilde{\Delta}_{F}\left(p_{f}^{2}\right)=\frac{1}{2} \int_{0}^{\infty} d T \frac{\left\langle p_{f}|U(T)| x_{i}\right\rangle}{\left\langle p_{f} \mid x_{i}\right\rangle}=-\frac{i}{p_{f}^{2}+m^{2}-i \varepsilon}
$$

in agreement with Eq. (1.5). Having reviewed the relationship between propagators and path integrals in a simple case, we now consider the extension to a scalar particle interacting with a gauge field.

\subsection{Scalar particle in an abelian background gauge field}

We consider a charged scalar particle in an abelian background gauge field. Such a system is described by the generating functional

$$
Z\left[J^{*}, J\right]=\int \mathcal{D} \phi^{*} \mathcal{D} \phi \exp \left[i \int d^{d} x\left(\phi^{*}\left(D_{\mu} D^{\mu}-m^{2}+i \varepsilon\right) \phi+J^{*} \phi+\phi^{*} J\right)\right],
$$

where $J, J^{*}$ are sources for the complex scalar field, and $D_{\mu}=\partial_{\mu}-i A_{\mu}$. By completing the square and defining $S=\left(-D_{\mu} D^{\mu}+m^{2}\right)$ we can write this as

$$
Z\left[J^{*}, J\right]=\int \mathcal{D} \phi^{*} \mathcal{D} \phi \exp \left[i \int d^{d} x\left(-\phi^{*}(S-i \varepsilon) \phi-J^{*}(S-i \varepsilon)^{-1} J\right)\right] .
$$

The propagator is given by the inverse of operator quadratic in $\phi, \phi^{*}$, which gives Eq. (1.4) as before. Using the fact that $p_{\mu}=-i \partial_{\mu}$, we can write the operator $S$ in normal form (i.e. with all momenta operators on the left-hand-side) as

$$
S=(p-A)^{2}+m^{2}=p^{2}-p \cdot A-A \cdot p+A^{2}+m^{2}=p^{2}-2 p \cdot A-i(\partial \cdot A)+A^{2}+m^{2} .
$$


Now defining the Hamiltonian operator $H=\frac{1}{2} S$ as before, one may carry out the manipulations of the previous section to obtain the first-quantized path integral representation of the evolution operator sandwiched between the external line position and momentum states

$$
\begin{gathered}
\left\langle p_{f}|U(T)| x_{i}\right\rangle=\int_{x(0)=x_{i}}^{p(T)=p_{f}} \mathcal{D} p \mathcal{D} x \exp \left[-i p(T) x(T)+i \int_{0}^{T} d t\left(p \dot{x}-\frac{1}{2}\left(p^{2}+m^{2}\right)+p \cdot A\right.\right. \\
\left.\left.+\frac{i}{2} \partial \cdot A-\frac{1}{2} A^{2}\right)\right] .
\end{gathered}
$$

This differs from the free particle case due to the presence of the gauge field in the exponent. When the strength of the gauge field is weak, the classical path of the emitting particle is well approximated by the free particle solution of Eq. $(1.14)^{4}$. One then finds

$$
\left\langle p_{f}|U(T)| x_{i}\right\rangle=e^{-i p_{f} x_{i}-i \frac{1}{2}\left(p_{f}^{2}+m^{2}\right) T} f(T),
$$

where

$$
\begin{gathered}
f(T)=\int_{x(0)=0}^{p(T)=0} \mathcal{D} p \mathcal{D} x \\
\quad \exp \left[i \int _ { 0 } ^ { T } d t \left(p \dot{x}-\frac{1}{2} p^{2}+\left(p_{f}+p\right) \cdot A\left(x_{i}+p_{f} t+x\right)\right.\right. \\
\left.\left.+\frac{i}{2} \partial \cdot A\left(x_{i}+p_{f} t+x\right)-A^{2}\left(x_{i}+p_{f} t+x\right)\right)\right] .
\end{gathered}
$$

Again the boundary conditions have again been made explicit, and we have dropped the primes on the quantities defined in Eq. (1.14).

\subsection{Spinor particle}

We now consider the case of an emitting fermion in the presence of a background gauge field. The system is described by the generating functional

$$
Z[\eta, \bar{\eta}]=\int \mathcal{D} \bar{\psi} \mathcal{D} \psi \exp \left[i \int d^{d} x(\bar{\psi}(\not D-m) \psi+\bar{\eta} \psi+\bar{\psi} \eta)\right]
$$

where $\bar{\eta}, \eta$ are Grassmann-valued source fields ${ }^{5}$. The momentum space propagator is then given by

$$
\Delta_{F}=\frac{1}{-i(\not D-m)}=(\not D+m) \frac{1}{i\left[(-i \not D)^{2}+m^{2}\right]} .
$$

Now we define $S=(-i \not D)^{2}+m^{2}$, by analogy with the scalar case. Using the standard trick

$$
\gamma^{\mu} \gamma^{\nu}=\frac{1}{2}\left\{\gamma^{\mu}, \gamma^{\nu}\right\}+\frac{1}{2}\left[\gamma^{\mu}, \gamma^{\nu}\right]
$$

\footnotetext{
${ }^{4}$ We will formalize this statement in section 2.2 when we discuss next-to-eikonal exponentiation.

${ }^{5}$ Recall we use the metric $(-,+,+,+)$ throughout.
} 
we can rewrite

$$
(-i \not D)^{2}+m^{2}=p^{2}-2 p \cdot A-i \partial \cdot A+A^{2}+m^{2}-\sigma^{\mu \nu} F_{\mu \nu}
$$

where $\sigma^{\mu \nu}=-\frac{i}{4}\left[\gamma^{\mu}, \gamma^{\nu}\right]$ are the generators of the Lorentz group, and $F_{\mu \nu}$ is the field strength tensor for the gauge field. Carrying out the path integral manipulations as in the scalar case yields

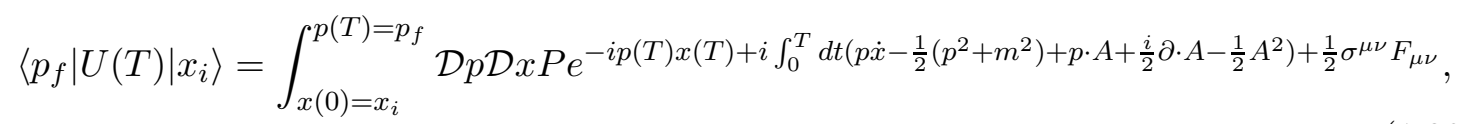

The representation (1.28) is almost identical to the case of a scalar eikonal line, apart from the coupling to the field strength tensor. This is not surprising, as it is well known $[14,29]$ that one can cast fermion actions into a second-order form that gives rise to scalar-like vertices supplemented by additional seagull vertices involving couplings to the field strength (see e.g. [30, 31]). The latter correspond physically to the magnetic moment of the spinning emitting particle, and thus have no analogue in the scalar case.

In this introduction, we have reviewed the representation of particle propagators, including the possible presence of an abelian background gauge field, as first-quantized path integrals. The phase space variables $x$ and $p$ correspond to the position and momentum of the emitting particle, and the classical path is interpreted as an eikonal line. In the following section we formalize these statements, and show how the above representations can be used to derive the exponentiation of soft radiative corrections.

\section{Soft emissions in scattering processes}

We now turn to the description of soft radiation from external lines, considering Green's functions having the form shown schematically in figure 4, and consisting of a hard interaction $H\left(x_{1}, \ldots, x_{n}\right)$ with external lines emerging at positions $\left\{x_{i}\right\}$. This is a sum of subdiagrams containing gauge boson modes of as yet unspecified momentum. Each external line has a propagator associated with it summing the effect of soft gauge boson emission, and we call such diagrams eikonally factorized.

This is based upon the general analysis of [32], which characterizes the regions of infrared sensitivity in Feynman diagrams in a number of scattering processes. The proof that soft radiation contributions exponentiate now amounts to showing two things. Firstly, that for the eikonally factorized diagrams defined above (and shown in Fig. 4), the contributions from soft radiation on the outgoing external lines exponentiates. Secondly, that all leading soft radiation terms originate from diagrams having this factorized structure. In this paper, we prove the first property and assume that the second indeed holds, as has been shown to be the case elsewhere at eikonal level. We return to the issue of corrections to the above factorized form (at NE level) in Sec. 2.4.

To make these statements more direct, we begin by separating the path integral over the gauge boson field into a product of integrals over hard and soft modes

$$
\int \mathcal{D} A^{\mu} \equiv \int \mathcal{D} A_{s}^{\mu} \mathcal{D} A_{h}^{\mu}
$$




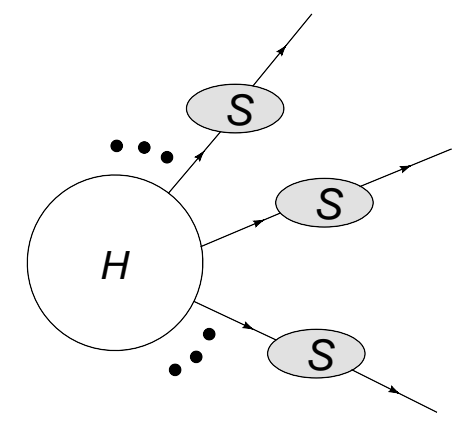

Figure 4: The factorized form of the Green's functions of Eq. (2.5), where $H$ is a hard interaction with $n$ outgoing external lines, and $S$ is a propagator for the eikonal particle in the presence of the background soft gauge field which, after the path integral over $A_{s}^{\mu}$, generates connections between the external lines.

The precise definition of $A_{s}^{\mu}$ and $A_{h}^{\mu}$ amounts to specifying a surface in the multi-boson momentum space that separates it into two distinct regions corresponding to soft and hard modes. Such a surface is, in general, very complicated [32]. Its precise definition does not concern us in what follows, where we characterize soft radiation by the fact that one may neglect the recoil of the eikonal particles ${ }^{6}$. However, the fact that such a surface exists allows us to introduce the factorization of Eq. (2.1), given that the path integral is a product of integrals over gauge fields of definite momentum.

The above separation is not gauge invariant, which can be easily seen as follows. Consider a given soft gauge field $A_{s}^{\mu}$, whose momentum modes live only in the soft region of momentum space. A general gauge transformation has the form

$$
A_{s}^{\mu}(x) \rightarrow A_{s}^{\mu}(x)+\partial^{\mu} \xi(x)
$$

for some function $\xi(x)$. Transforming to momentum space, $\xi(x)$ may well have momentum modes defined in the hard region of momentum space, and thus the transformed gauge field will have in general both soft and hard components. Instead, both the soft and hard gauge fields obey a restricted gauge invariance given by the momentum space analogue of Eq. (2.2)

$$
A_{s, h}^{\mu}(k) \rightarrow A_{s, h}^{\mu}(k)+k^{\mu} \xi^{\prime}(k) .
$$

Here $\xi^{\prime}(k)$ is non-zero only if $k$ is in the soft and hard regions for $A_{s}^{\mu}$ and $A_{h}^{\mu}$ respectively.

We now formally define the hard interaction as

$$
\begin{aligned}
H\left(x_{1}, \ldots, x_{n}\right)= & \int \mathcal{D} A_{h}^{\mu} \mathcal{D} \phi \mathcal{D} \phi^{*} \frac{1}{i^{n}} \frac{\delta}{\delta J\left(y_{1}\right)} \ldots \frac{\delta}{\delta J\left(y_{n}\right)}\left\langle y_{1}|S-i \epsilon| x_{1}\right\rangle \ldots\left\langle y_{n}|S-i \epsilon| x_{n}\right\rangle \\
& \times \exp \left[i S\left[\phi, \phi^{*}, A^{\mu}\right]+i \int d^{d} x\left(J(x) \phi^{*}(x)+J(x) \phi^{*}(x)\right)\right] .
\end{aligned}
$$

This is analogous to the expression for a Green's function, except for the fact that the path integral over soft gauge field modes $A_{s}^{\mu}$ has yet to be performed. Also, the factors

\footnotetext{
${ }^{6}$ We will consider corrections to this idea when discussing next-to-eikonal exponentiation in section 2.2 .
} 
$\left\langle y_{i}|S-i \epsilon| x_{i}\right\rangle$ (i.e. inverse propagators for the particle in the background of the soft gauge field) truncate the external legs of the Green's function.

We now define the further quantity

$$
G\left(p_{1}, \ldots, p_{n}\right)=\int \mathcal{D} A_{s}^{\mu} H\left(x_{1}, \ldots, x_{n}\right)\left\langle p_{1}\left|(S-i \varepsilon)^{-1}\right| x_{1}\right\rangle \ldots\left\langle p_{n}\left|(S-i \varepsilon)^{-1}\right| x_{n}\right\rangle,
$$

where a propagator factor has been associated with each external line, and the path integral over $A_{s}^{\mu}$ inserted. This latter integral does two things. Firstly, it generates all possible subgraphs within the hard interaction $H$ (i.e. such that there are $n$ external lines emerging at 4-positions $\left\{x_{i}\right\}$ ), containing both soft and hard gauge boson modes. Secondly, it produces soft radiation, both real and virtual, from the external lines. This is shown schematically in figure 4, where real and soft radiation is included in the soft blobs attached to each external line. One thus sees that $G$ is a full Green's function of the theory, written in eikonally factorized form. Note that the propagator for the emitting particle in the presence of the background soft gauge field is removed in Eq.( 2.4), and replaced in Eq.( 2.5) with the propagator sandwiched between states of given initial position and final momentum.

To obtain the contribution to the scattering amplitude from the function $G\left(p_{1}, \ldots, p_{n}\right)$, one must truncate each external propagator. That is, for external leg $i$ one multiplies by a factor $p_{i}^{2}+m^{2}$ to take account of the fact that the line is external and thus has no (free) propagator attached. In principle one must also divide by the residue of the scalar propagator, arising from renormalization of the scalar field. However, this residue is unity due to the absence of self-interactions for the eikonal particle, and also the fact that the gauge field is treated as a background. As is clear from Eq. (2.5), one may treat each external line separately. Using the representation (1.21), one can rewrite each external line contribution as

$$
\begin{aligned}
i\left(p_{f}^{2}+m^{2}\right)\left\langle p_{f}\right| & -i(S-i \varepsilon)^{-1}\left|x_{i}\right\rangle=i\left(p_{f}^{2}+m^{2}\right) \frac{1}{2} \int_{0}^{\infty} d T e^{-i p_{f} x_{i}-i \frac{1}{2}\left(p_{f}^{2}+m^{2}-i \varepsilon\right) T} f(T) \\
= & -e^{-i p_{f} x_{i}} \int_{0}^{\infty} d T\left(\frac{d}{d T} e^{-i \frac{1}{2}\left(p_{f}^{2}+m^{2}\right) T}\right)\left(e^{-\frac{1}{2} \varepsilon T} f(T)\right) \\
= & -e^{-i p_{f} x_{i}}\left(-f(0)-\int_{0}^{\infty} d T e^{-i \frac{1}{2}\left(p_{f}^{2}+m^{2}\right) T}\left(\frac{d}{d T} e^{-\frac{1}{2} \varepsilon T} f(T)\right)\right) \\
= & -e^{-i p_{f} x_{i}}\left(-f(0)-\int_{0}^{\infty} d T e^{-i \frac{1}{2}\left(p_{f}^{2}+m^{2}\right) T} \frac{d}{d T} f(T)\right)
\end{aligned}
$$

In the last step we have taken the limit $\varepsilon \rightarrow 0$. At this point one can let $p_{f}$ approach its mass shell and obtain the simple result

$$
i\left(p_{f}^{2}+m^{2}\right)\left\langle p_{f}\left|-i(S-i \varepsilon)^{-1}\right| x_{i}\right\rangle=e^{-i p_{f} x_{i}} f(\infty) .
$$

The limit $T \rightarrow \infty$ of $f(T)$ in Eq. (2.7) allows us to simplify the expression for $f$ in Eq. (1.22) by performing the Gaussian integral over $p$. After shifting the integration variable $p \rightarrow p+A$ the result is a path integral over $x$ only

$$
f(\infty)=\int_{x(0)=0} \mathcal{D} x e^{i \int_{0}^{\infty} d t\left(\frac{1}{2} \dot{x}^{2}+\left(p_{f}+\dot{x}\right) \cdot A\left(x_{i}+p_{f} t+x(t)\right)+\frac{i}{2} \partial \cdot A\left(x_{i}+p_{f} t+x\right)\right)} .
$$


Thus, the eikonally factorized contribution to the scattering amplitude for a charged scalar in an abelian background field takes the form

$$
S\left(p_{1}, \ldots, p_{n}\right)=\int \mathcal{D} A_{s}^{\mu} H\left(x_{1}, \ldots, x_{n}\right) e^{-i p_{1} x_{1}} f_{1}(\infty) \ldots e^{-i p_{n} x_{n}} f_{n}(\infty) e^{i S\left[A_{s}\right]} .
$$

with $f(\infty)$ given by Eq. (2.8), and the label of each $f(\infty)$ indicates the particular external line. Also we have explicitly factored out the action for the soft gauge field, which remains after the path integrals over the particle and hard gauge fields. This form (2.9) will now enable us to find all-order expressions for these amplitudes.

As a simple one-dimensional path integral, it can be further manipulated using simple classical methods. The strictest approximation is to neglect the fluctuations $x(t)$ and $p(t)$. This is equivalent to the eikonal approximation in Feynman diagrams, and one sets $x=0, \dot{x}=0, p=0$ and as well as neglecting the $\partial \cdot A$ and $A^{2}$ terms in Eq. (1.21). One then finds an Aharanov-Bohm-like phase factor for the straight line trajectory

$$
f(\infty) \propto e^{i \int d x \cdot A(x)} .
$$

Inserting this result into the path integral (2.9) where we integrate over soft gauge field fluctuations the Wilson lines, being linear in the soft gauge field $A_{s}^{\mu}$, act as a collection of classical source terms for the soft $A$-field, distributed along the classical trajectory.

\subsection{Eikonal exponentiation}

Now that we have established that in the eikonal approximation the soft radiation is de-

scribed by a Wilson line we can analyze what happens in perturbation theory. Let us consider, without loss of generality, an external line created at $x_{i}=0$ and in direction $n^{\mu} \equiv p_{f}^{\mu}$. Then Eq. (2.10) becomes

$$
\exp \left[i \int_{0}^{\infty} d t n^{\mu} A_{\mu}(n t)\right] .
$$

This can be written, after a Fourier transform to momentum space, as

$$
i \int_{0}^{\infty} d t n^{\mu} A_{\mu}(n t)=-\int \frac{d^{d} k}{(2 \pi)^{d}} \frac{n^{\mu} \tilde{A}_{\mu}(k)}{n \cdot k} .
$$

Note that this is invariant under rescalings of the eikonal momentum $n^{\mu}$. As seen above, this acts as a source term for the soft gauge field when the path integral over $A_{s}^{\mu}$ is performed. It can be represented as a 1-photon vertex with the momentum space Feynman rule

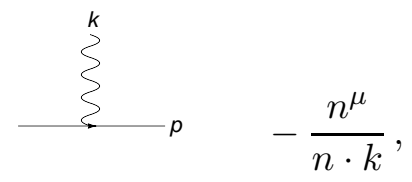

where momentum $k$ flows into the vertex. The path integral over the soft gauge field generates all possible diagrams connecting numbers of source vertices. By the usual rules of quantum field theory, one finds connected and disconnected diagrams, which in this case 


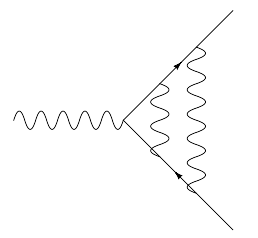

Figure 5: Example of a disconnected subdiagram between two outgoing external lines, to be compared with the connected subdiagrams of figure 1.

connect the external lines given that the source vertices lie along the latter. The collection of all diagrams exponentiates in terms of connected diagrams.

To illustrate this further, consider the case of a hard interaction with two outgoing eikonal lines, an example of which is shown in figure 1. There one sees a number of connected subdiagrams connecting the external lines. One also finds disconnected diagrams, such as that shown in figure 5. However, given the usual property of exponentiation of disconnected diagrams in quantum field theory, one has:

$$
\sum G=\exp \left[\sum G_{c}\right]
$$

The sum on the left is over all subdiagrams $G$, while the sum on the right is over all connected diagrams $G_{c}$.

We have thus succeeded in showing that the exponentiation of soft radiative corrections in the eikonal limit can be related to the exponentiation of disconnected diagrams. We now consider what happens when next-to-eikonal corrections are considered.

\subsection{Next-to-eikonal exponentiation}

The analysis of the previous section relied on the fact that the factors $f(\infty)$ describing soft radiation from the external lines are written as path integrals in $x$. This allowed the straightforward interpretation of the eikonal limit as the limit in which the emitting particle follows a classical free path. However, this identification also allows one to go easily beyond the eikonal approximation. If the emitted radiation is soft but with non-negligible momentum, the classical path is still a good approximation to the equations of motion for the eikonal particle, and one can examine deviations from the straight-line path in a systematic expansion. Given that this does not affect the interpretation of gauge boson emission vertices in terms of disconnected subdiagrams, one still expects such corrections to exponentiate.

To formalize this argument, we reconsider the external line given by Eq. (1.14), where again we take $x_{i}=0$ without loss of generality. Given that the external eikonal particles have light-like momenta, one may write $p_{j}=\lambda n_{j}$, where $n_{j}^{2}=0$. Then Eq. (2.8) becomes

$$
f(\infty)=\int_{x(0)=0} \mathcal{D} x \exp \left[i \int_{0}^{\infty} d t\left(\frac{1}{2} \dot{x}^{2}+(\lambda n+\dot{x}) \cdot A(\lambda n t+x)+\frac{i}{2} \partial \cdot A(\lambda n t+x)\right)\right] .
$$


One now clearly sees that in the limit $\lambda \rightarrow \infty$, one may neglect all terms involving $x, \dot{x}$ and $\partial \cdot A$, leaving precisely the eikonal approximation discussed in the previous section. That is, fluctuations about the classical free path are suppressed by inverse powers of $\lambda$. By expanding in $\lambda$, one keeps the first subleading corrections to the eikonal approximation, i.e. corresponding to the next-to-eikonal (NE) limit.

For subsequent purposes it is more convenient to rescale the time variable $t \rightarrow t / \lambda$, so that Eq. (2.15) becomes

$$
f(\infty)=\int_{x(0)=0} \mathcal{D} x \exp \left[i \int_{0}^{\infty} d t\left(\frac{\lambda}{2} \dot{x}^{2}+(n+\dot{x}) \cdot A(n t+x)+\frac{i}{2 \lambda} \partial \cdot A(n t+x)\right)\right] .
$$

The first term in the exponent is now $\sim \mathcal{O}(\lambda)$, but gives rise to a propagator for $x(t)$ which is $\mathcal{O}\left(\lambda^{-1}\right)$ by virtue of being the inverse of the quadratic operator in $x(t)$. The remaining terms generate effective vertices for soft gauge boson emission in the NE limit, which one can again interpret as source terms for the soft gauge field. Thus, following the reasoning in the previous section, one finds that these NE corrections exponentiate as before, i.e. one has:

$$
f(\infty)=\exp \left[\sum G_{c}^{x}\right]
$$

where $G_{c}^{x}$ are connected (through $x$ propagators) diagrams along external lines, and located on the latter by vertices derived by a systematic expansion of Eq.(2.16) in $\lambda^{-1}$. At LO, one recovers the eikonal approximation of the previous section. To obtain the NE approximation one must gather all terms $\sim \mathcal{O}\left(\lambda^{-1}\right)$, which can be described as follows.

Firstly, NE graphs must have at most one propagator for the emitting particle $x(t)$, due to its being $\mathcal{O}\left(\lambda^{-1}\right)$ as remarked above. There is also a NE vertex originating from the term in $\partial \cdot A$, and a given $\mathrm{NE}$ graph containing such a vertex must then contain no propagator factors for $x(t)$. We examine in detail the NE Feynman rules that result from Eq. (2.16) in appendix B, and show that they agree with the results one obtains in standard perturbation theory after expanding to NE order. The advantage of the above representation, however, is that exponentiation of these corrections is manifest.

\subsection{Exponentiation for spinor particles}

We have so far only considered the case of a scalar eikonal particle. For emitting fermions (with a similar expression for combinations of fermions and antifermions etc.), we write the definition of the hard interaction as

$$
\begin{aligned}
H\left(x_{1}, \ldots, x_{n}\right)=\int \mathcal{D} A_{h}^{\mu} \mathcal{D} \psi \mathcal{D} \bar{\psi} \frac{1}{i^{n}} \frac{\delta}{\delta \bar{\eta}\left(y_{1}\right)} \ldots \frac{\delta}{\delta \bar{\eta}\left(y_{n}\right)}\left\langle y_{1}\left|S_{0}-i \epsilon\right| x_{1}\right\rangle \ldots\left\langle y_{n}\left|S_{0}-i \epsilon\right| x_{n}\right\rangle \\
\quad \times \exp \left[i S\left[\psi, \bar{\psi}, A^{\mu}\right]+i \int d^{d} x(\bar{\eta}(x) \psi(x)+\bar{\psi} \eta(x))\right]
\end{aligned}
$$

where $S_{0}-i \epsilon$ is the free fermion inverse propagator. The eikonally factorized Green's

function has the same form as before (Eq. (2.5)), where the propagator in the presence of the background gauge field is given by Eqs. (1.28). Truncating the external lines of the full 
Green's function ${ }^{7}$, one finds that the eikonally factorized scattering amplitudes are given by the same expression Eq. (2.9), but where the external line factor is now:

$$
\begin{aligned}
f(\infty)=\int_{x(0)=0} \mathcal{D} x \exp \left[i \int _ { 0 } ^ { \infty } d t \left(\frac{\lambda}{2} \dot{x}^{2}+(n+\right.\right. & \dot{x}) \cdot A(n t+x) \\
& \left.\left.+\frac{i}{2 \lambda} \partial \cdot A(n t+x)+\frac{1}{2 \lambda} \sigma^{\mu \nu} F_{\mu \nu}\right)\right],
\end{aligned}
$$

and we have rescaled the time variable $t \rightarrow t / \lambda$ as before. The proof of exponentiation up to NE order proceeds directly as in the scalar case, except for the additional magnetic moment vertex which, although absent in the scalar case, does nothing to invalidate the proof. Note that, due to suppression by $\lambda$, the additional vertex is indeed of NE order, as expected given that in the strict eikonal limit, radiation is insensitive to the spin of the emitting particle.

One may worry about ordering of Dirac matrices when the exponential of Eq. (2.19) is expanded. However, this is not an issue due to the fact that the magnetic moment vertex is NE and thus occurs in each diagram only once at this order.

The preceding analysis has shown that in eikonally factorized Green's functions, soft gauge boson corrections exponentiate up to NE order. This is not yet a proof that such corrections exponentiate in matrix elements themselves, which include Green's functions not having an eikonally factorized structure. At strictly eikonal level (as is well known), one may in fact ignore contributions from diagrams which are not eikonally factorized. At $\mathrm{NE}$ order, however, contributions arise from diagrams in which a soft emission connects an external line with the hard interaction. This is the subject of the following section.

\subsection{Low's theorem}

In the previous section we have demonstrated exponentiation for next-to-eikonal photon emissions from external lines. That is, the exponentiation holds for scattering amplitudes having the eikonally factorized form of figure 4. However, at NE order there are also corrections to the exponentiation arising from soft gluon emissions which land on an external line, having originated from inside the hard interaction. A given matrix element then has the schematic form (up to next-to-eikonal level):

$$
\mathcal{M}=\exp \left[\mathcal{M}^{\mathrm{E}}+\mathcal{M}^{\mathrm{NE}}\right]\left(1+\mathcal{M}_{r}\right)
$$

Here $\mathcal{M}^{\mathrm{E}, \mathrm{NE}}$ collect the eikonal and next-to-eikonal diagrams from eikonally factorized Green's functions respectively, and $\mathcal{M}_{r}$ is a remainder term which does not exponentiate, and contains NE contributions from diagrams such as that shown in figure 6 . In what follows, we refer to emissions from within the hard interaction as internal emissions, and those originating from external lines as external. Diagrams with internal emissions have been studied before in the literature. It has been shown for a fixed number of scalar external

\footnotetext{
${ }^{7}$ In the spinor case we defined the evolution operator $U(T)$ as involving only the denominator of Eq. (1.25). The leftover factor in the numerator indeed combines correctly with the inverse free propagator to give a factor $p_{f}^{2}+m^{2}$ as in the scalar case of Eq. (2.6).
} 


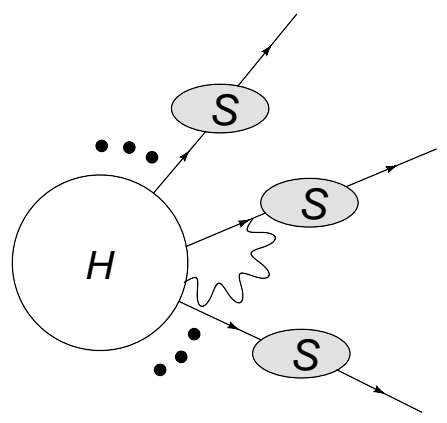

Figure 6: An example of a diagram which contributes to the remainder factor $\mathcal{M}_{r}$ of Eq. (2.20). Such contributions are formed by taking an eikonally factorized Green's function, and adding a soft gluon emission which lands on an external line, but originates from inside the hard interaction.

lines that, up to NE order, the sum of diagrams containing a soft emission (internal or external) can be related to the scattering amplitude with no emissions [33]. This result is known as Low's theorem, and was generalized to the case of spinor external lines in [34], an extension known as the Low-Burnett-Kroll theorem. Generalization to higher orders was considered in [35]. The fact that graphs with an extra emission can be related simply to those without an emission means that, although the remainder term does not have a formal exponential structure, it has an iterative form to all orders in perturbation theory. In this section we discuss these properties in the path integral formalism adopted in this paper, in order to complete our discussion of NE exponentiation. This also allows for a generalization of the ideas presented in $[33,34,35]$.

Our starting point is the expression for the $n$-particle scattering amplitude (see also Eq. (2.9))

$S\left(p_{1}, \ldots, p_{n}\right)=\int \mathcal{D} A_{s} H\left(x_{1}, \ldots, x_{n} ; A_{s}\right) e^{-i p_{1} x_{1}} f\left(x_{1}, p_{1} ; A_{s}\right) \ldots e^{-i p_{n} x_{n}} f\left(x_{1}, p_{1} ; A_{s}\right) e^{i S\left[A_{s}\right]}$,

where we have explicitly indicated the dependence of the external leg factors (Eq. (2.16)) on the soft gauge field. We also now consider the fact that the external lines are produced at 4 -positions $x_{i} \neq 0$ i.e.

$$
\begin{array}{r}
f\left(x_{i}, p_{f}, A_{s}\right)=\int_{x(0)=x_{i}}^{p(\infty)=p_{f}} \mathcal{D} x \exp \left[i \int _ { 0 } ^ { \infty } d t \left(\frac{\lambda}{2} \dot{x}^{2}+q_{i}(n+\dot{x}) \cdot A\left(x_{i}+n t+x\right)\right.\right. \\
\left.\left.+\frac{i}{2 \lambda} q_{i} \partial \cdot A\left(x_{i}+p t+x\right)\right)\right] .
\end{array}
$$

We have also indicated the dependence of the hard part on the soft photon field $A_{s}$ and rescaled this field so as to explicitly display the dependence on the eikonal particle's electric charge $q_{i}$. Furthermore, we drop the subscript $s$ on the gauge field in what follows, and leave implicit the path integrals over the external line 4-positions $x_{i}$.

Both $H$ and the $f$ 's depend on the soft gauge field $A$. As discussed in section 2 , the separation between soft and hard gauge modes leaves a residual gauge invariance, given 
by the usual form of Eq. (2.2), but where the function $\Lambda(x)$ has only soft modes when transformed to momentum space. It is implicitly assumed that this in the case in the following. Under such a transformation, the external line factors transform as

$$
f\left(x_{i}, p_{f} ; A\right) \rightarrow f\left(x_{i}, p_{f} ; A+\partial \Lambda\right)=e^{-i q \Lambda\left(x_{i}\right)} f\left(x_{i}, p_{f} ; A\right)
$$

which follows from the definition of Eq. (2.7).

In order for the path integral to remain invariant under the family of gauge transformations given by Eq. (2.2), the hard function $H$ must transform as

$$
H\left(x_{1}, \ldots, x_{n} ; A\right) \rightarrow H\left(x_{1}, \ldots, x_{n} ; A+\partial \Lambda\right)=H\left(x_{1}, \ldots, x_{n} ; A\right) e^{i q_{1} \Lambda\left(x_{1}\right)+\ldots+i q_{n} \Lambda\left(x_{n}\right)} .
$$

We can now use the gauge invariance to relate diagrams with soft emissions from inside the hard interaction to similar diagrams with no emission, as follows. First, one may expand both sides of Eq. (2.24) to first order in $A$ and in $\Lambda$, which gives

$$
\begin{aligned}
H\left(x_{1}, \ldots, x_{n}\right)+\int d^{d} x H^{\mu}\left(x_{1}, \ldots, x_{n} ; x\right)\left(A_{\mu}(x)+\partial_{\mu} \Lambda(x)\right) & \\
H\left(x_{1}, \ldots, x_{n}\right) & +\int d^{d} x H^{\mu}\left(x_{1}, \ldots, x_{n} ; x\right) A_{\mu}(x) \\
& +i \int d^{d} x\left(H\left(x_{1}, \ldots, x_{n}\right) \sum_{j}^{n} q_{j} \delta\left(x-x_{j}\right)\right) \Lambda(x) .
\end{aligned}
$$

When the path integral over the soft gauge field is performed, $H^{\mu}\left(x_{1}, \ldots, x_{n} ; x\right)$ generates hard interactions which a single soft photon emission (with Lorentz index $\mu$ ). Because $\Lambda(x)$ is arbitrary we infer

$$
-\partial_{\mu} H^{\mu}\left(x_{1}, \ldots, x_{n} ; x\right)=i H\left(x_{1}, \ldots, x_{n}\right) \sum_{j}^{n} q_{j} \delta\left(x-x_{j}\right),
$$

where we have integrated by parts on the left hand side of Eq. (2.25). In momentum space this becomes

$$
-k_{\mu} H^{\mu}\left(p_{1}, \ldots, p_{n} ; k\right)=\sum_{j}^{n} q_{j} H\left(p_{1}, \ldots, p_{j}+k, \ldots, p_{n}\right) .
$$

One can expand this up to first order in $k$ to obtain

$$
-k_{\mu} H^{\mu}\left(p_{1}, \ldots, p_{n} ; k\right)=\sum_{j}^{n} q_{j} k_{\mu} \frac{\partial}{\partial p_{j_{\mu}}} H\left(p_{1}, \ldots, p_{n}\right),
$$

where the zeroth order term on the right hand side vanishes due to charge conservation $\sum_{j} q_{j}=0$. Now, because $k^{\mu}$ is an arbitrary soft momentum, one may write

$$
H^{\mu}\left(p_{1}, \ldots, p_{n} ; k\right)=-\sum_{j}^{n} q_{j} \frac{\partial}{\partial p_{j_{\mu}}} H\left(p_{1}, \ldots, p_{n}\right) .
$$


This simply relates internal emission from the hard interaction with the same hard interaction but with no emission. For a simple example of how this works using more traditional methods, we refer the reader to appendix C.

In section 2 we ignored the fact that the external lines emerge at positions $x_{i}$ which are integrated over and thus in general non-zero. Taking this into account also leads to corrections of NE order (and beyond), which enter the above remainder term $\mathcal{M}_{r}$. To see this, we write the eikonal one-photon source term as:

$$
-q \int \frac{d^{d} k}{(2 \pi)^{d}} \frac{n \cdot A(k)}{n \cdot k} e^{i x \cdot k}
$$

This can be expanded as

$$
-q \int \frac{d^{d} k}{(2 \pi)^{d}}(1+i x \cdot k) \frac{n \cdot A(k)}{n \cdot k},
$$

where the first bracketed term corresponds to the eikonal approximation, and the second term (involving the factor $x \cdot k$ ) is suppressed by one power of momentum and is thus a $\mathrm{NE}$ correction. We now combine these terms with the factors of Eq. (2.29), and up to NE order the scattering amplitude is given by a sum over all such corrections, where each NE factor occurs at most once. One then finds

$$
\begin{array}{r}
S\left(p_{1}, \ldots, p_{n}\right)=\int \mathcal{D} A\left[\left(-\sum_{j}^{n} q_{j} \frac{\partial}{\partial p_{j}} H\left(p_{1}, \ldots, p_{n}\right)\right) \int \frac{d^{d} k}{(2 \pi)^{d}} A_{\mu}(k)+\right. \\
\left.\int d x_{1}^{d} \ldots d x_{n}^{d} H\left(x_{1}, \ldots, x_{n}\right)\left(\sum_{j} q_{j} \int \frac{d^{d} k}{(2 \pi)^{d}}\left(-i x_{j} \cdot k\right) \frac{n \cdot A(k)}{n \cdot k}\right) e^{-i x_{1} \cdot p_{1}-\ldots-i x_{n} \cdot p_{n}}\right] \\
\\
f\left(0, p_{1} ; A\right) \ldots f\left(0, p_{n} ; A\right),
\end{array}
$$

where we have explicitly instated the integrals over the initial positions of the external lines $\left\{x_{i}\right\}$. Performing these integrals, the scattering amplitude is given by

$$
\begin{array}{r}
S\left(p_{1}, \ldots, p_{n}\right)=\int \mathcal{D} A\left[\int \frac{d^{d} k}{(2 \pi)^{d}} \sum_{j}^{n} q_{j}\left(\frac{n_{j}^{\mu}}{n_{j} \cdot k} k_{\nu} \frac{\partial}{\partial p_{j_{\nu}}}-\frac{\partial}{\partial p_{j_{\mu}}}\right) H\left(p_{1}, \ldots, p_{n}\right) A_{\mu}(k)\right] \\
\times f\left(0, p_{1} ; A\right) \ldots f\left(0, p_{n} ; A\right) .
\end{array}
$$

Some comments are in order regarding the form and interpretation of this result. The external line factors $f\left(0, p_{i} ; A\right)$ contain exponentiated eikonal and NE terms, as discussed previously. Corrections to the NE exponentiation then arise due to the bracketed prefactor in Eq. (2.33), which contains a sum over different possible NE corrections. Such corrections contribute to the remainder term $\mathcal{M}_{r}$ in Eq. (2.20), and do not exponentiate. However, Eq. (2.33) shows that they can be obtained as derivatives of the hard interaction with no soft emissions. Thus, the remainder term has an iterative structure to all orders in perturbation theory.

For scattering amplitudes, one may summarize this as follows. Leading eikonal logarithms arising from soft gluon emission exponentiate. NE logarithms do not exponentiate, 
but can be separated into the sum of a series which does exponentiate, and a remainder sum which does not exponentiate, but is obtainable in principle to all orders in the coupling constant.

To further clarify the above formulae, it is instructive to consider the case where the hard interaction $H$ is a scalar. Then it can only depend on Lorentz invariant products of momenta. The derivatives with respect to the 4-momenta in Eq. (2.33) can be reexpressed in terms of derivatives with respect to products of 4-vectors (corresponding to Mandelstam invariants in the hard scattering process). One may verify that derivatives w.r.t. $p_{i}^{2}$ vanish, and in the case of two external lines $(n=2)$ there is only one scalar $p_{1} \cdot p_{2}$. Eq. (2.33) then becomes

$$
\begin{aligned}
& S\left(p_{1}, \ldots, p_{n}\right)=\int \mathcal{D} A {\left[\int \frac{d^{d} k}{(2 \pi)^{d}}\left(\frac{n_{1}^{\mu}\left(k \cdot n_{2}-k \cdot n_{1}\right)}{n_{1} \cdot k}+\frac{n_{2}^{\mu}\left(k \cdot n_{1}-k \cdot n_{2}\right)}{n_{2} \cdot k}\right)\right.} \\
&\left.\times \frac{\partial}{\partial\left(p_{1} \cdot p_{2}\right)} H\left(p_{1}, \ldots, p_{n}\right) A_{\mu}(k)\right] f\left(0, p_{1} ; A\right) \ldots f\left(0, p_{n} ; A\right) .
\end{aligned}
$$

This is precisely the form one expects based on a conventional Feynman diagram treatment (see appendix C), and one may interpret the bracketed factor in Eq. (2.34) as an extra vertex describing soft emission from within the hard interaction.

\section{Non-abelian gauge theory}

So far we have considered an abelian background gauge field. In the case where the gauge field is non-abelian, the derivation of the scattering amplitude for eikonally factorized diagrams proceeds similarly to Sec. 2 . Here we consider the simple case of a hard interaction with two outgoing external lines. That is, the analogue of Eq. (2.9) can be written

$$
S\left(p_{1}, p_{2}\right)=\int \mathcal{D} A_{s}^{\mu} H^{i_{1} i_{2}}\left(x_{1}, x_{2}\right) e^{-i p_{1} x_{1}} f_{1}^{i_{1} j_{1}}(\infty) e^{-i p_{2} x_{2}} f_{2}^{i_{2} j_{2}}(\infty) e^{i S\left[A_{s}\right]} .
$$

Here $\left\{i_{k}\right\}$ and $\left\{j_{k}\right\}$ are indices in the fundamental representation of the gauge group, such that the outgoing particles have indices $\left\{j_{k}\right\}$ and summation over repeated color indices is implied. The external line factors $f^{i_{k} j_{k}}(\infty)$ have the form

$$
f^{i_{1} j_{1}}(\infty)=\left[\int_{x(0)=x_{i}} \mathcal{D} x \mathcal{P} e^{i \int_{0}^{\infty} d t\left(\frac{1}{2} \dot{x}^{2}+\left(p_{f}+\dot{x}\right) \cdot A\left(x_{i}+p_{f} t+x(t)\right)+\frac{i}{2} \partial \cdot A\left(x_{i}+p_{f} t+x\right)\right)}\right]^{i_{1} j_{1}},
$$

i.e. similar to before, but matrix-valued in color space due to the exponent being linear in the non-abelian gauge field $A^{\mu}=A_{A}^{\mu} t^{A}$, where $t^{A}$ is a generator of the gauge group. Furthermore, there is a path ordering of the color matrices along the external line. As before, the external line factors act as source terms for the soft gauge field when the path integral over $A_{s}^{\mu}$ is performed. However, it is no longer immediately clear that the soft corrections exponentiate. In the abelian case, the exponentiation of soft gauge boson corrections was identified with the exponentiation of disconnected diagrams between sources. Crucial to the combinatorics of this result is the fact that in the abelian case, the source 
terms commute with each other. This is no longer true in the non-abelian case, due to the matrix valued nature of the source terms, and also the path ordering of the exponential in Eq. (3.2). We will see, however, that it is still possible to address exponentiation in the non-abelian case, by rephrasing the problem using the replica trick of statistical physics (see appendix A for another application i.e. the proof of the exponentiation of disconnected diagrams in field theory). One can then write the scattering amplitude in a form such that extra structure emerges in the exponent, whereby the contraction of soft gluon emissions between eikonal lines gives rise to a exponentiating subset of diagrams. These can then be identified with the webs of $[11,10]$.

To simplify the discussion, we first restrict ourselves to the strict eikonal limit. Furthermore, we consider the case of a hard interaction with the color singlet structure

$$
H^{i_{1} i_{2}}\left(x_{1}, x_{2}\right)=H\left(x_{1}, x_{2}\right) \delta^{i_{1} i_{2}},
$$

where $\delta^{i_{1} i_{2}}$ is the Kronecker symbol. Such a structure arises in interactions where e.g. an incoming color singlet particle gives rise to the pair production of two hard final charged scalars (the scalar analogue of $e^{+} e^{-}$pair production by a virtual photon), as shown in figure 2. Given that, up to the NE corrections discussed in section 2.4, one may consider the external lines as being created at $x=0$, one may take the hard interaction outside the path integral over $A_{s}$ in Eq. (3.1) to obtain (in this case)

$$
S\left(p_{1}, p_{2}\right)=H\left(p_{1}, p_{2}\right) \int \mathcal{D} A_{s}^{\mu} f_{1}^{i j_{1}}(\infty) f_{2}^{i j_{2}}(\infty) e^{i S\left[A_{s}\right]}
$$

Here $S\left[A_{s}\right]$ is the action for the soft gauge field which is independent of the emitting particles. The product of external line factors, suppressing momentarily the color indices, is given by

$$
f_{1}(\infty) f_{2}(\infty)=\left[\mathcal{P} e^{i \int d x_{1} \cdot A\left(x_{1}\right)}\right]\left[\mathcal{P} e^{i \int d x_{2} \cdot A\left(x_{2}\right)}\right]
$$

The first factor is a Wilson line parametrized by $x_{1}(s)$, where $s=-t$ increases along the direction of the charge flow, with $-\infty<s<0$. The second factor is a Wilson line parametrized by $x_{2}(s)$, with $s=t$ and $0<s<\infty$. One may combine these into a single curve given by

$$
x(s)=\left\{\begin{array}{lr}
x_{1}(s), & -\infty<s<0 ; \\
x_{2}(s), & 0 \leq s<\infty .
\end{array}\right.
$$

Due to the path ordering in the definition of the Wilson line, one has the property

$$
\left[\mathcal{P} e^{i \int d x_{1} \cdot A\left(x_{1}\right)}\right]\left[\mathcal{P} e^{i \int d x_{2} \cdot A\left(x_{2}\right)}\right]=\mathcal{P} e^{i \int d x \cdot A(x)},
$$

so that, for the simple interaction considered here, one may combine the two external line factors into the single factor

$$
f(\infty)=f_{1}(\infty) f_{2}(\infty)=\mathcal{P} e^{i \int d x \cdot A(x)} .
$$

The scattering amplitude of Eq. (3.4) is now given by:

$$
S\left(p_{1}, p_{2}\right)=H\left(p_{1}, p_{2}\right) \mathcal{F},
$$


where

$$
\mathcal{F}=\int \mathcal{D} A_{s}^{\mu} f(\infty) e^{i S\left[A_{s}\right]}
$$

We now consider the quantity

$$
\mathcal{F}^{N}=\left[\int \mathcal{D} A_{1}^{\mu} f^{(1)}(\infty) e^{i S\left[A_{1}\right]}\right] \ldots\left[\int \mathcal{D} A_{N}^{\mu} f^{(N)}(\infty) e^{i S\left[A_{N}\right]}\right] .
$$

Here $\left\{A_{i}^{\mu}\right\}$ are $N$ replicas of the soft gauge field (we have dropped the subscript $s$ for brevity), and $S\left[A_{i}\right]$ the action for the $i^{t h}$ replica. One has a different external line factor $f^{(i)}$ for each replica field. Combining the path integrals using $\mathcal{D} A^{\mu} \equiv \prod_{i} \mathcal{D} A_{i}^{\mu}$, one can rewrite Eq. (3.11) as

$$
\mathcal{F}^{N}=\int \mathcal{D} A^{\mu} f^{(1)}(\infty) \ldots f^{(N)}(\infty) e^{i S\left[A_{1}\right]+\ldots+i S\left[A_{N}\right]} .
$$

The physical interpretation of this quantity is as follows. The external line factors, as in the abelian case, contain sources for the gauge field. In this case, they generate diagrams containing any mixture of the $N$ replica gauge fields, which span the external lines of the hard interaction (which in this case have become a single external line). Each of the vertices for the emission of a gauge field replica has a non-trivial color structure, such that neither the external factors $f^{(i)}$ nor the vertices they give rise to commute. However, by definition one has:

$$
\mathcal{F}^{N}=1+N \log (\mathcal{F})+\mathcal{O}\left(N^{2}\right) .
$$

It follows that, if one can extract a term in Eq. (3.12) that is linear in the number $N$ of replica fields, one has

$$
\mathcal{F}=\exp \left[\sum W\right]
$$

where the sum is over all diagrams $W$ that contribute at $\mathcal{O}(N)$. Crucially, we will find that not all diagrams in the theory have terms of $\mathcal{O}(N)$, so one recovers the property of exponentiation of soft radiative corrections in terms of a subset of diagrams with certain properties. The diagrams in this case will still contain replica fields. However, given that the gauge group of the replicated theory is the same as that in the standard theory, it must be true that the color structures of the subdiagrams which exponentiate are the same in the two theories.

We now describe how to isolate the term linear in $N$ in Eq. (3.12). The product of external line factors has the form:

$$
f^{(1)}(\infty) \ldots f^{(N)}(\infty)=\mathcal{P} \exp \left[\int d x \cdot A_{1}(x)\right] \ldots \mathcal{P} \exp \left[\int d x \cdot A_{N}(x)\right] .
$$

Ideally we want to write this as a single path-ordered exponential, so that one can identify the usual rules of perturbation theory. This can be achieved by writing Eq. (3.15) in the following form:

$$
\prod_{i=1}^{N} \mathcal{P} \exp \left[\int d x \cdot A_{i}(x)\right]=\mathcal{R} \mathcal{P} \exp \left[\sum_{i=1}^{N} \int d x \cdot A_{i}(x)\right]
$$




$\begin{array}{lllll}t_{0} & t_{0}^{\prime} & t_{1} & t_{2} & t_{1}^{\prime} \\ 2 & 2 & 2 & 2 & 2 \\ 2 & 2 & 2 & 2 & 2 \\ 2 & 2 & 2 & 2 & 2\end{array}$

Figure 7: Example of an radiative corrections generated by the source terms of Eq. (3.17), where two gauge boson replicas are shown. Each set of replica emissions is time ordered, such that (in this case) $t_{2}>t_{1}>t_{0}$ and $t_{1}^{\prime}>t_{0}^{\prime}$.

where we have introduced the replica ordering operator $\mathcal{R}$, defined such that

$$
\mathcal{R}\left[A_{i}(x) A_{j}(y)\right]=\left\{\begin{array}{ll}
A_{i}(x) A_{j}(y), & i \leq j \\
A_{j}(y) A_{i}(x), & i>j
\end{array},\right.
$$

with obvious generalization to higher numbers of operators. That is, $\mathcal{R}$ orders any product of matrix-valued fields into a sequence of increasing replica number. Note that the resulting product is no longer strictly time ordered, although the matrix fields of any given replica number remain time ordered.

As before (and by analogy with conventional Feynman perturbation theory for nonAbelian gauge fields), the single exponent in Eq. (3.17) acts as a collection of sources for the soft gauge field. The path integration over the soft gauge field generates diagrams containing multiple replica emissions along the eikonal line, where the replica numbers are not necessarily ordered along the line (see figure 7 ). However, the expression for a given diagram, as dictated by the source terms arising from Eq. (3.16), involves replica ordered products of operators, each of which involves a color matrix. Thus, the color structure associated with each diagram is not the same as that which would result from conventional perturbation theory, but rather that associated with the given replica-ordered product of matrix-valued fields. The subset of diagrams which exponentiates then has a modified color structure, as is known to be the case for webs $[10,11,12]$. To see which diagrams $W$ actually contribute in Eq. (3.14), one must consider contracting gluons emitted from two or more vertices. Given that the gauge field replicas do not interact with each other (i.e. are only tangled through color structure), one can clearly only contract gluons which have the same replica number $i$ and adjoint color index $A$. Here we consider this up to $\mathcal{O}\left(A_{\mu}^{4}\right)$ in the scattering amplitude (i.e. up to two gluon lines). Firstly, we need only consider contributions from vertices on different segments of the combined Wilson line $x(t)$, as those on the same segment ultimately give contributions proportional to (at least in covariant gauges) $p_{1}^{2}=0$ or $p_{2}^{2}=0$. Also, each diagram has a multitude of similar diagrams obtained by permuting the replica labels. The operator $\mathcal{R}$ for each diagram orders the color matrices in the form

$$
\left[t_{1}^{A_{1}} \ldots t_{1}^{A_{n_{1}}}\right] \ldots\left[t_{N}^{B_{1}} \ldots t_{N}^{B_{n_{N}}}\right]
$$

i.e. a product of strings of color matrices, with one string for each replica (if present), and $n_{i}$ matrices for replica $i$. Given that the replicas do not interact with each other, the color 


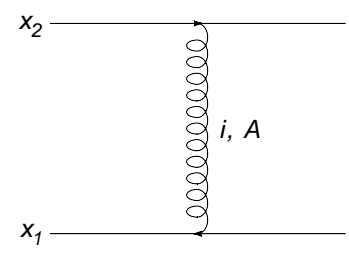

(a)

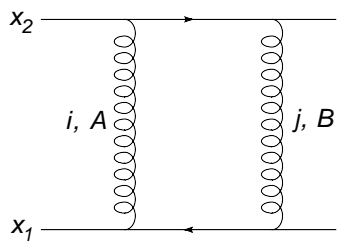

(b)

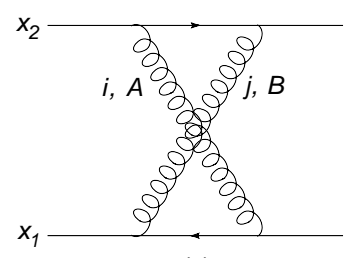

(c)

Figure 8: Diagrams that potentially contribute to the exponentiated contribution to the scattering amplitude in the case of two external lines connected by a color singlet structure, for one and two gluon emissions. In fact, only (a) and (c) contribute as discussed in the text.

indices of each string in Eq. (3.18) are contracted independently of the other strings. That is, the color factor for each diagram has the form

$$
\prod_{i=1}^{N} K_{i}^{A_{1} \ldots A_{n_{i}}}\left[t_{1}^{A_{1}} \ldots t_{1}^{A_{n_{i}}}\right]
$$

where $K_{i}^{A_{1} \ldots A_{n_{i}}}$ is a combination of factors involving $f^{A B C}$ and $\delta^{A B}$ which implements the color contractions for replica $i$ in the diagram being considered. Each of the strings of color matrices corresponding to a given replica number in Eq. (3.19) has two color indices in the fundamental representation, thus by Schur's Lemma must be proportional to the identity. Hence, the color factor of a complete diagram in the replica ordered perturbation theory is the product of the individual color factors associated with the subdiagrams formed from each replica separately. Furthermore, the ordering of the factors in Eq. (3.18) is unimportant, so that the color factors associated with the set of diagrams obtained from a given diagram merely by permuting replica numbers are the same. There are ${ }_{N} P_{m}=$ $N ! /(N-m)$ ! such permutations, where $m$ is the number of different replica species present in the diagram.

For one gluon emission, there is only one possible diagram, shown in figure 8(a). There is a sum over the replica number of the exchanged gluon, so that this diagram is clearly proportional to $N$, denoting a color structure that exponentiates. The color factor of this diagram is

$$
t^{A} t^{A}=C_{F}
$$

where $t^{A}$ is a generator in the fundamental representation of the gauge group, and $C_{F}$ the relevant Casimir invariant. Note that this is the same as the color factor in conventional perturbation theory, although things become more complicated when more than one gluon is involved.

For two gluon emission, one has the two diagrams shown in Fig. 8(b,c). For each of these, one must consider separately the cases where $i=j$ and $i \neq j$, because of the fact that the replica ordering operator $\mathcal{R}$ acts differently in the two cases.

For Fig. 8(b), in the case where $i=j$ one has a color factor

$$
t_{j}^{B} t_{i}^{A} t_{i}^{A} t_{j}^{B}=C_{F}^{2},
$$




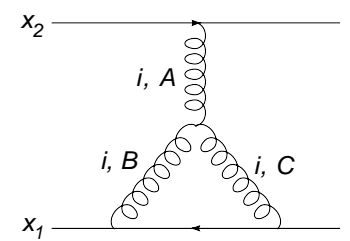

(a)

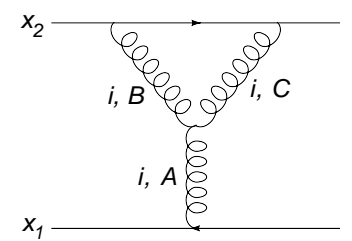

(b)

Figure 9: Diagrams contributing to the exponentiated scattering amplitude at $\mathcal{O}\left(\alpha_{S}^{4}\right)$, and involving the 3 -gluon vertex.

where we have explicitly indicated which color matrix is associated with each replica. When $i \neq j$, the color matrices in Eq. (3.22) get reordered by the $\mathcal{R}$ operator i.e. one has

$$
t_{i}^{A} t_{i}^{A} t_{j}^{B} t_{j}^{B}=C_{F}^{2}
$$

for $i<j$, with a similar expression for $i>j$ (but where $i$ and $j$ are interchanged). The color factors of these diagrams are the same, and thus one may combine the results for $i=j$ and $i \neq j$. Then one sees that the contribution from Fig. (8)(b) is $\mathcal{O}\left(N^{2}\right)$.

For Fig. 8(c), the $i=j$ case has the color factor

$$
t_{i}^{A} t_{j}^{B} t_{i}^{A} t_{j}^{B}=C_{F}^{2}-\frac{C_{F} C_{A}}{2} .
$$

When $i \neq j$ one has

$$
t_{i}^{A} t_{i}^{A} t_{j}^{B} t_{j}^{B}=C_{F}^{2} .
$$

Note that the color factors for $i=j$ and $i \neq j$ are now different, such that the two cases do not combine to give a term $\mathcal{O}\left(N^{2}\right)$. There are $N$ diagrams where $i=j$, and ${ }_{N} P_{2}=N(N-1)$ diagrams where $i \neq j$. Thus the term linear in $N$ has a color factor:

$$
N\left(C_{F}^{2}-\frac{C_{F} C_{A}}{2}\right)+(-N) C_{F}^{2}=N\left(-\frac{C_{F} C_{A}}{2}\right) .
$$

The above discussion can be summarized as follows. Up to two gluon emissions, a subset of diagrams exponentiates. Namely, the one gluon emission diagram of Fig. 8(a), and the crossed gluon diagram of Fig. 8(c). Fig. 8(b) does not contribute due to being $\mathcal{O}\left(N^{2}\right)$, and Fig. 8(c) has a color factor which differs from that of conventional perturbation theory, and indeed is precisely the modified color factor associated with the known webs of $[10,11,12]$.

Note at this order in $\alpha_{S}$ one also has diagrams containing gluon self-interactions, of which there are two possibilities, shown in Fig. 9. There are $N$ such diagrams in each case, so that they also exponentiate (i.e. are webs) with a color factor equal to the ordinary one.

We now consider the generalization of the above remarks to higher orders in perturbation theory. The subdiagrams which exponentiate can be characterized by the fact that they are two-eikonal irreducible. That is, one cannot disconnect each diagram by cutting eikonal lines in two places. This property is well-known $[11,10]$, but we prove it in the following using the methods outlined above. 


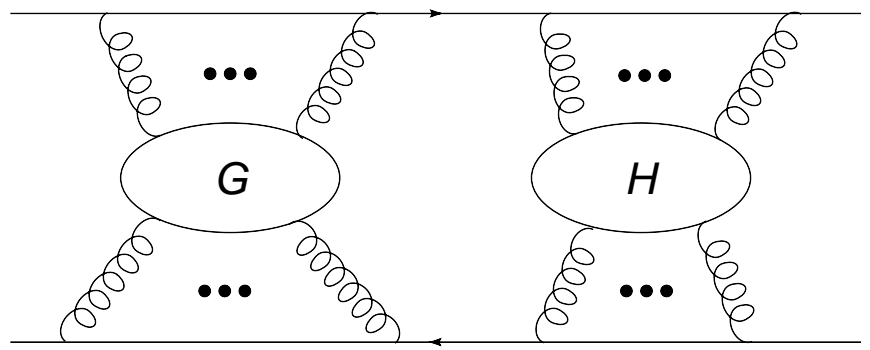

Figure 10: A two-eikonal reducible diagram, in the simplest case of two disconnected components with respect to the external lines.

Consider the general two-eikonal reducible diagram shown in Fig. 10 (where each of the subdiagrams could itself be reducible). We focus on a given gauge boson replica $i$ in subdiagram $H$ in Fig. 10, and consider first the case where subdiagram $G$ contains no gauge bosons with replica number $i$. According to the Feynman rules of the replica-ordered perturbation theory described above, the diagram of Fig. 10 then gives a color factor

$$
K_{i}^{A_{1} \ldots A_{n} B_{1} \ldots B_{m}}\left[t_{H}^{A_{1}} \ldots t_{H}^{A_{n}}\right]\left[t_{H}^{B_{m}} \ldots t_{H}^{B_{1}}\right] \times \prod_{j \neq i} C(j)
$$

where $C(j)$ is the color factor associated with replica $j$, and $K_{i}^{A_{1} \ldots A_{n} B_{1} \ldots B_{m}}$ is the color contraction factor for replica $i$ introduced above. That is, $\prod C(j)$ contains the results of all contractions in $G$ as well as those of $H$ that do not involve replica number $i$. The indices $\left\{A_{k}\right\}$ and $\left\{B_{k}\right\}$ denote emissions from the lower and upper eikonal lines respectively. Now consider the case where $G$ as well as $H$ contains emissions with replica number $i$. Then (due to the reducible structure) one can write $K_{i}$ as a product of factors $S_{G_{i}}, S_{H_{i}}$ for each subdiagram, so that the color factor associated with Fig. 10 is

$$
\begin{aligned}
& K_{G_{i}}^{A_{n_{H}+1} \ldots A_{n} B_{m_{H}+1} \ldots B_{m}} S_{H_{i}}^{A_{1} \ldots A_{n_{H}} B_{1} \ldots B_{m_{H}}}\left[t_{H}^{A_{1}} \ldots t_{H}^{A_{n_{H}}}\right] \\
& \times \quad\left[t_{G}^{A_{n_{H}+1}} \ldots t_{G}^{A_{n}}\right]\left[t_{G}^{A_{m}} \ldots t_{G}^{A_{m_{H}+1}}\right]\left[t_{H}^{B_{m_{H}}} \ldots t_{H}^{B_{1}}\right] \times \prod_{j \neq i} C(j) .
\end{aligned}
$$

We may use the fact that

$$
K_{G_{i}}^{A_{n_{H}+1} \ldots A_{n} B_{m_{H}+1} \ldots B_{m}}\left[t_{G}^{A_{n_{H}+1}} \ldots t_{G}^{A_{n}}\right]\left[t_{G}^{A_{m}} \ldots t_{G}^{A_{m_{H}+1}}\right] \propto I
$$

where $I$ is the identity in color space. This follows given that the contribution in color space from a given replica in a disconnected subdiagram $G$ has two indices in the fundamental representation. By Schur's Lemma, this must be proportional to the identity i.e. the only possible two-index invariant tensor. We may then rewrite Eq. (3.27) as

$$
\begin{aligned}
& K_{G_{i}}^{A_{n_{H}+1} \ldots A_{n} B_{m_{H}+1} \ldots B_{m}} K_{H_{i}}^{A_{1} \ldots A_{n_{H}} B_{1} \ldots B_{m_{H}}}\left[t_{H}^{A_{1}} \ldots t_{H}^{A_{n_{H}}}\right] \\
& \times \quad\left[t_{H}^{B_{m_{H}}} \ldots t_{H}^{B_{1}}\right]\left[t_{G}^{A_{n_{H}+1}} \ldots t_{G}^{A_{n}}\right]\left[t_{G}^{A_{m}} \ldots t_{G}^{A_{m_{H}+1}}\right] \times \prod_{j \neq i} C(j) .
\end{aligned}
$$


This has the same color structure as would arise if one were considering a different replica number in $G$ than has been considered in $H$. I.e. one can absorb all $G$-dependent factors into the product $\prod C(j)$, such that Eq. (3.29) is then the same as Eq. (3.26). The color structures of the subdiagrams are thus independent, and it follows that the number of ways of forming the total diagram in Fig 10 is the product of the number of ways of forming the individual subdiagrams. For each subdiagram this is at least $\propto N$, such that the contribution from diagrams which are two-eikonal reducible is at least $\propto N^{2}$ (in general, $\propto N^{M}$, where $M$ is the number of disconnected subdiagrams). Thus, two-eikonal reducible diagrams do not exponentiate.

In fact, one can proceed further and obtain a general solution for the color factors of the diagrams to all orders. Consider a given diagram consisting of $n_{c}$ connected pieces (i.e. gluons connected by self interactions or fermion bubbles). For each such diagram, we then consider the set $\{P\}$ of partitions. These are sets containing a number $n(P)$ of subgraphs $g$, each of which contains only one replica (see Fig. 11). Permuting the replica numbers (but keeping the subgraphs $g$ intact) corresponds to the same partition (see Fig. 12), such that there $\operatorname{are}_{N} P_{n(P)}$ distinct diagrams in each partition, each of which has the same color factor

$$
\prod_{g \in P} C(g)
$$

where $C(g)$ is the color factor associated with subgraph $g$. The total color factor of the complete diagram is now given by

$$
\sum_{P}{ }_{N} P_{n(P)} \prod_{g \in P} C(g)
$$

i.e. one sums over all possible partitions, each of which has a color factor given by Eq. (3.30), and weighted by the number of diagrams represented by each partition. The only dependence on the replica number resides in the factor ${ }_{N} P_{n(P)}$, which has the form

$$
{ }_{N} P_{n(P)}=(-1)^{n(P)-1}(n(P)-1) ! N+\mathcal{O}\left(N^{2}\right) .
$$

The contribution from a given complete diagram $G$ which is linear in $N$ is thus given by

$$
\bar{C}(G)=\sum_{P}(-1)^{n(P)-1}(n(P)-1) ! \prod_{g \in P} C(g) .
$$

The bar on the left hand side denotes the fact that this is the modified color factor associated with $G$, rather than the color factor one would obtain in conventional perturbation theory (i.e. without the $\mathcal{R}$ operator). The color factors on the right hand side, $C(g)$, are the ordinary color factors associated with each replica subgraph $g$. Eq. (3.33) is a closed form solution for the modified color factor associated with any given diagram, in that modified color factors only appear on the left-hand side. It has the property, as it must do from the above considerations, of being zero if $G$ is not a web. As a simple example (already encountered above), consider the diagram of Fig. 8(c). There are two possible partitions. Either the two gluons have the same replica number, i.e. one subgraph with 


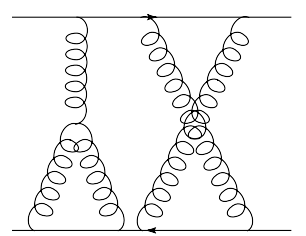

(a)

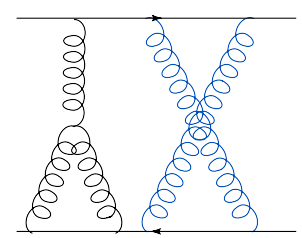

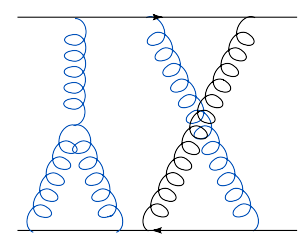

(b)

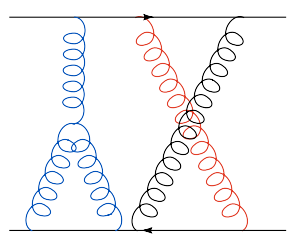

Figure 11: Examples of (a) a diagram containing 3 connected pieces; (b) three possible partitions generated by this diagram, where colors represent distinct replica numbers. Permuting colors gives the same partition.
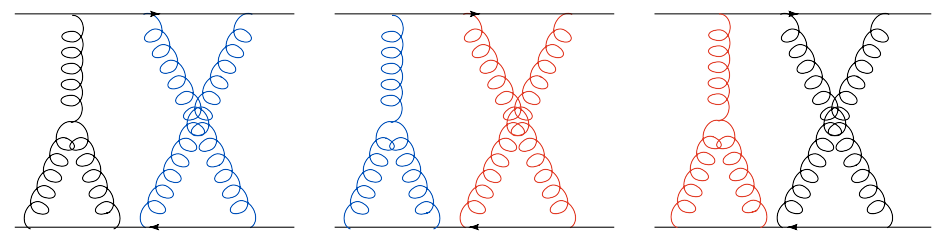

Figure 12: Examples of diagrams in the same partition, where colors represent distinct replica numbers.

color factor $C(X)$, or they have different color factors i.e. two subgraphs with total color factor $C(\mid) C(\mid)$. Then Eq. (3.33) gives a total modified color factor

$$
\bar{C}(X)=C(X)-C(\mid) C(),
$$

which gives $-\frac{1}{2} C_{F} C_{A}$ as required.

To further clarify the discussion, we consider here a pair of three loop examples. We consider first the diagram represented in the above shorthand notation by $X$. From Eq. (3.33), one has schematically

$$
\begin{aligned}
\bar{C}(X) & =C(X)-2 C(\mid) C(X)-C(\mid) C(||)+2 C()^{3} \\
& =C(X)-2 \bar{C}() \bar{C}(X)-\bar{C}(\mid)^{3}
\end{aligned}
$$

where in the second line we have used the fact that $\bar{C}(\mid)=C(\mid)$ and $\bar{C}(\mathrm{X})=C(\mathrm{X})-C(\mid)^{2}$. The second line then agrees with the modified color factor given in $[10,11]$.

Our second example is $\mathrm{X}$, for which one has

$$
\bar{C}(\mid X)=C(\mid X)-C(\mid) C(X) .
$$

Reducibility implies $C(\mid X)=C() C(X)$, and thus $\bar{C}(\mid X)=0$, as expected for a non-web.

To summarize, the above replica trick has allowed us to determine the subset of diagrams which exponentiate in non-abelian theory i.e. the diagrams which exponentiate are those which have a term linear in the number of replicas $N$. This is related to the original gauge theory (with no replicas) as follows. Firstly, the replica gluons all have the same self-interactions and scalar-gluon interactions, but do not interact with each other. 
Thus in any diagram one can replace replica gluons with original gluons to yield the same kinematic result. Then the color weights of the exponentiating diagrams are precisely those found above i.e. modified with respect to the original theory. The structure of two-eikonal irreducible diagrams with modified color factors is precisely that of web exponentiation, described in $[11,13]$.

The above discussion proceeds similarly in the case of fermionic emitting particles, given that the gauge boson emission vertices which distinguish scalar from fermion emitters only appear at NE order. We discuss subleading corrections in the next section.

\subsection{Non-abelian exponentiation at NE order}

The significance of the above derivation of web exponentiation in terms of the path integral method is that one may then easily extend the analysis to NE order, using the $\lambda$-scaling technique discussed in section 2.2. We have already shown that a subset of NE corrections exponentiate in the abelian case, and that eikonal corrections in the non-abelian case exponentiate. Thus, it is not surprising that a subset of non-abelian NE terms exponentiates.

In this section we again consider the simple case of two external lines emerging from a hard interaction which has a color singlet structure. Then the scattering amplitude factorizes as in Eq. (3.10), but where the external line factor is given by

$$
\begin{aligned}
f(\infty)=\int_{x(0)=0} \mathcal{D} x \mathcal{P} \exp \left[i \int _ { 0 } ^ { \infty } d t \left(\frac{\lambda}{2} \dot{x}^{2}+(n\right.\right. & +\dot{x}) \cdot A(n t+x) \\
& \left.\left.+\frac{i}{2 \lambda} \partial \cdot A(n t+x)-\frac{1}{2 \lambda} \sigma^{\mu \nu} F_{\mu \nu}\right)\right],
\end{aligned}
$$

One next performs the path integral in $x$ (as detailed for the abelian case in appendix B), which gives

$$
\begin{aligned}
f(\infty)=\mathcal{P} & \exp \left[i \int_{0}^{\infty} d t n \cdot A(n t)-\frac{1}{2 \lambda} \int_{0}^{\infty} d t \partial \cdot A(n t)\right. \\
& +\frac{1}{2} \int_{0}^{\infty} d t \int_{0}^{\infty} d t^{\prime}\left\langle x^{\mu}(t) \dot{x}^{\nu}\left(t^{\prime}\right)\right\rangle A_{\mu}(n t) A_{\nu}\left(n t^{\prime}\right) \\
& -\int_{0}^{\infty} d t \int_{0}^{\infty} d t^{\prime}\left\langle\dot{x}^{\mu}(t) x^{\alpha}\left(t^{\prime}\right)\right\rangle n^{\nu} A_{\mu}(n t) \partial_{\alpha} A_{\nu}\left(n t^{\prime}\right) \\
& -\frac{1}{2} \int_{0}^{\infty} d t \int_{0}^{\infty} d t^{\prime}\left\langle x^{\alpha}(t) x^{\beta}\left(t^{\prime}\right)\right\rangle n^{\mu} n^{\nu} \partial_{\alpha} A_{\mu}(n t) \partial_{\beta} A_{\nu}\left(n t^{\prime}\right) \\
& \left.+\frac{i}{2} \int_{0}^{\infty} d t n^{\mu}\left\langle x^{\nu}(t) x^{\alpha}(t)\right\rangle \partial_{\nu} \partial_{\alpha} A_{\mu}(n t)\right] .
\end{aligned}
$$

For reasons that will become clear, we have stayed in position space in the exponent. 


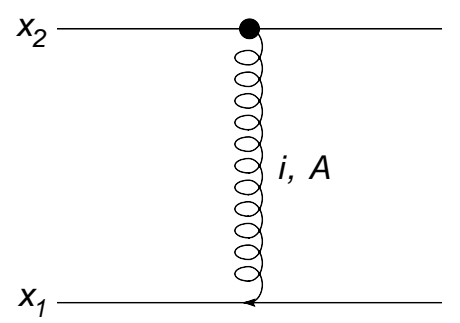

(a)

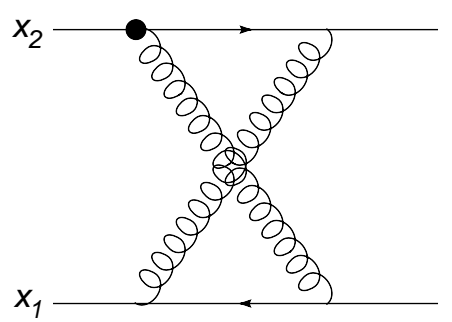

(c)

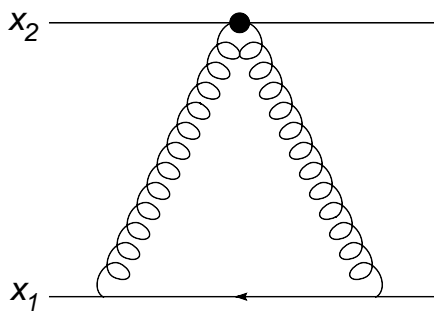

(b)

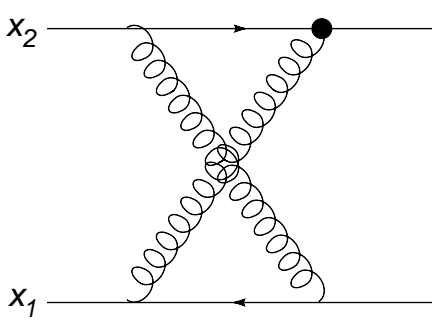

(d)

Figure 13: Webs involving local vertices occurring at NE order, where $\bullet$ represents a vertex of NE order. Additional diagrams arise by reflecting each diagram shown about the horizontal axis.

Inserting the correlators of the $x$ fields (see Eqs. (B.18)), Eq. (3.38) becomes:

$$
\begin{aligned}
f(\infty)=\mathcal{P} & \exp \left[i \int_{0}^{\infty} d t n \cdot A^{A}(n t) t^{A}-\frac{1}{2 \lambda} \int_{0}^{\infty} d t \partial \cdot A^{A}(n t) t^{A}\right. \\
+ & \frac{1}{\lambda} \int_{0}^{\infty} d t \eta^{\mu \nu} A_{\mu}^{A}(n t) A_{\nu}^{B}(n t) \frac{1}{2}\left\{t^{A}, t^{B}\right\}+\frac{i}{2 \lambda} \int_{0}^{\infty} d t n^{\mu} \partial^{\nu} \partial_{\nu} A_{\mu}^{A}(n t) t^{A} \\
& -\frac{1}{\lambda} \int_{0}^{\infty} d t \int_{0}^{t} d t^{\prime} \eta^{\mu \alpha} n^{\nu} A_{\mu}^{A}(n t) \partial_{\alpha} A_{\nu}^{B}\left(n t^{\prime}\right) t^{A} t^{B} \\
& \left.-\frac{1}{\lambda} \int_{0}^{\infty} d t \int_{0}^{t} d t^{\prime} t^{\prime} n^{\mu} n^{\nu} \partial_{\alpha} A_{\mu}^{A}(n t) \partial_{\beta} A_{\nu}^{B}\left(n t^{\prime}\right) t^{A} t^{B}\right] .
\end{aligned}
$$

Here we have explicitly factored out color matrices from the non-abelian gauge fields. Note that path ordering (i.e. time ordering in this case) has appeared in some of the terms in the exponent, due to the $\Theta$ function occurring in the correlators of Eq. (B.18). There are two types of vertex occurring in Eq. (3.39) - those that depend on a single time (in the first two lines), and those that depend on two different times (in the third and fourth lines). Of the former type, there are one gluon vertices and a two gluon vertex. For these vertices, the arguments of the previous section (involving the replica trick) carry forward with minimal modification, and one has eikonal exponentiation up to NE webs, where each NE vertex must occur only once per diagram. The additional webs that appear at two gluon order are shown in figure 13. At higher orders, the NE webs again have the property of being two-external-line irreducible. The time ordered vertices in the third line of Eq. (3.39) generate diagrams such as those shown in figure 14, which involve correlated emissions from different positions on the external line, but where additional eikonal emissions may occur in between. One must then sum over all possible correlations (pairs of gluons). There are 


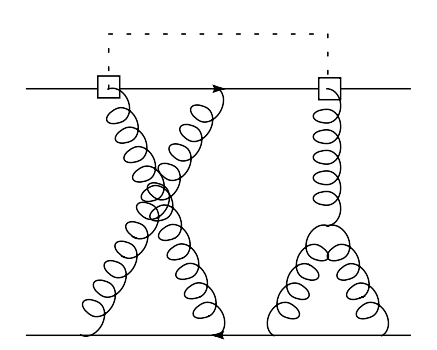

Figure 14: Example of a web involving the NE vertex (denoted here by $\square$ ), which correlates gluons emitted from different positions on the external line. As shown here, further eikonal emissions may occur between the correlated NE emissions.

two types of such diagrams. Firstly, diagrams whose structure is such that they form a web at eikonal level (i.e. are two-eikonal irreducible in that case). The time ordered vertices then implement correlations between pairs of gluons in the same web. Secondly, one has diagrams that would be two-eikonal reducible at eikonal level (i.e. are a product of webs), but which become irreducible at NE level due to correlations between gluons in separate webs (e.g. Fig. 14). The sum over all such diagrams then enters the exponent of Eq. (3.14). To summarize, the set of NE corrections resulting from non-abelian gauge boson emission outside of the hard interaction exponentiate. The exponent involves a subset of diagrams, NE webs, some of which are more complicated in structure than their eikonal equivalents. However, they still share the property of being two-external-line irreducible.

In the case of a spinor emitting particle, the additional vertex

$$
\frac{i}{2 \lambda} \int_{0}^{\infty} \sigma^{\mu \nu} F_{\mu \nu}
$$

appears in the exponent of Eq. (3.37) i.e. the non-abelian analogue of Eq. (B.30). This gives rise to both a one- and a two-gluon vertex, derived from the position space expressions

$$
\begin{array}{r}
\frac{i}{\lambda} \int_{0}^{\infty} \partial_{\mu} A_{\nu}^{A}(t) \sigma^{\mu \nu} t^{A} ; \\
\frac{1}{2} \int_{0}^{\infty} \sigma^{\mu \nu} A_{\mu}^{A}(t) A_{\nu}^{b}\left[t^{A}, t^{B}\right](t) .
\end{array}
$$

Both of these vertices depend on a single time, and thus are handled similarly to those occurring in Eq. (3.39). Note that the two-gluon vertex Eq. (3.42) has no abelian analogue, which can be seen from the fact that the commutator $\left[t^{A}, t^{B}\right]$ vanishes for an abelian gauge field.

Some comments are in order regarding the color factors associated with the NE webs. We note that the derivation of the formula for modified color factors in the eikonal case (Eq. (3.33)) is independent of the eikonal approximation. Thus, it also applies also in the NE case, where the color factors $C(j)$ associated with each subgraph involving replica number $j$ are the normal color factors one obtains using the NE Feynman rules.

The above discussion shows that, for the simple hard interaction considered above, a subset of corrections exponentiates up to NE order i.e. those associated with soft gauge 
boson emission outside the hard interaction. As in the abelian case, one still has to worry about corrections due to eikonal emissions from within the hard interaction (which give rise to the remainder term in Eq. (2.20)). This is the subject of the next section.

\subsection{Internal emissions of non-abelian gauge fields}

In the abelian case, we identified a subset of NE corrections which exponentiate (i.e. those arising from soft gauge boson emissions outside of the hard interaction). There were then remainder terms, which did not have an exponential structure but could be obtained iteratively to all orders in the perturbation expansion. Here we briefly discuss how this structure can be generalized to the case of a non-abelian gauge field. As in the previous section, we consider the case where the matrix element for the hard interaction with no internal emissions has a color singlet structure, with two outgoing eikonal lines.

We begin with the non-abelian analogue of the abelian gauge transformation law of Eq. (2.24), which is

$$
f\left(x_{i}, p_{f} ; A_{\mu}\right) \rightarrow f\left(x_{i}, p_{f}, U A_{\mu} U^{-1}-i U \partial_{\mu} U^{-1}\right)=U\left(x_{i}\right) f\left(x_{i}, p_{f} ; A_{\mu}\right)
$$

for an external particle, and

$$
f\left(x_{i}, p_{f} ; A_{\mu}\right) \rightarrow f\left(x_{i}, p_{f}, U A_{\mu} U^{-1}-i U \partial_{\mu} U^{-1}\right)=f\left(x_{i}, p_{f} ; A_{\mu}\right) U^{-1}\left(x_{i}\right)
$$

for an external antiparticle, where $U(x)=\exp \left(i \theta^{A}(x) t^{A}\right)$, and the coupling constant $g$ of the non-abelian gauge field has been absorbed in $A$. The hard interaction (in the two eikonal line, color singlet case considered in the previous sections) then transforms as

$$
H\left(x_{1}, x_{2}, A\right) \rightarrow H\left(x_{1}, x_{2}, U A_{\mu} U^{-1}-i U \partial_{\mu} U^{-1}\right)=U\left(x_{2}\right) H\left(x_{1}, x_{2}\right) U^{-1}\left(x_{1}\right) .
$$

Expanding this to first order in $A$ and $\theta^{a}(x)$, and using the fact that the latter is arbitrary gives the analogue of Eq. (2.26)

$$
-\partial_{\mu} H^{\mu}\left(x_{1}, x_{2} ; x\right) t^{A}=i H\left(x_{1}, x_{2}\right) \sum_{j} g_{j} \delta\left(x-x_{j}\right) t^{A},
$$

where $g_{j}=+1$ for an eikonal particle, and -1 for an antiparticle. This has the same interpretation as in the abelian case i.e. the amplitude for an internal emission is related to the amplitude with no such emission. In the non-abelian case, the amplitude for internal emission is proportional to the color matrix $t^{A}$, as indeed it must be from group theory considerations (the quantity $H^{\mu}$ has one adjoint index and two fundamental indices).

As in the abelian case, Eq. (3.46) can be interpreted as an extra vertex for soft gluon emission. This vertex is located on the Wilson line at $t=0$ i.e. where the eikonal segments $x_{1}$ and $x_{2}$ meet. There are terms (also by analogy with the abelian case of Sec. 2.4) which correct for the fact that the external lines do not originate from $x=0$. However, these also take the form of an additional vertex localized at $t=0$. The diagrams containing these additional vertices do not necessarily exponentiate (as before), and form a remainder term analogously to that of Eq. (2.20). 
Thus, the all-order structure of matrix elements up to NE order is conceptually equivalent to the abelian case. One has a subset of NE corrections which exponentiate (i.e. $\mathrm{NE}$ webs), and a set of corrections which form a remainder term which mixes with the exponentiated NE corrections. The remainder term does not necessarily have a simple structure, but at least has an iterative structure due to the fact that diagrams with no internal emission are sufficient to generate higher order diagrams involving an internal emission.

\section{Discussion}

In this paper, we have considered the issue of soft gauge boson corrections for matrix elements in abelian and non-abelian gauge theories from a path integral point of view. This involves considering factorized diagrams involving hard interactions with a given number of external lines, each of which emits further soft radiation. The propagator for each external line can then be cast into a first quantized path integral representation, where the integral is a sum over paths for the emitting particle. This path integral can be performed by expanding about the classical straight line path for the emitter, which corresponds to systematic corrections to the eikonal approximation. The scattering amplitude then factorizes into a hard interaction plus factors for each external line which act as source terms for the soft gauge field $A_{s}^{\mu}$, where the sources are placed along the external lines. In the abelian case, exponentiation of eikonal corrections then follows from the usual exponentiation of disconnected diagrams in quantum field theory. Furthermore, a subset of next-to-eikonal corrections can also be shown to exponentiate, and a set of effective Feynman rules for radiation in the NE limit is obtained.

The case of a non-abelian gauge field is more complicated, but can be analyzed using the replica trick, in which one considers an ensemble of $N$ gauge fields. Diagrams which have a term linear in $N$ then exponentiate, and crucially only a subset of diagrams in the theory have such a property. We considered the simple case of two external lines, connected by a hard interaction with color singlet structure. Then the diagrams which exponentiate contain sources arising from the replica ordered perturbation theory arising from Eq. (3.16). Those which contribute at $\mathcal{O}(N)$ have the property of being two-external line irreducible, and also have (in general) color factors which differ from those of the corresponding diagrams in the original theory. These diagrams are then precisely the webs of $[11,13]$. As in the abelian case, a subset of NE corrections also exponentiates, and the exponent contains a sum of webs up to NE level.

In both the abelian and non-abelian cases, there are NE corrections which do not exponentiate, and which form a remainder term such that the total matrix element (up to NE order) has the form shown in Eq. (2.20). These terms are associated with Low's theorem, and the relevant diagrams involve contractions between eikonal photons or gluons on the external lines, and a NE vertex localized at the cusp at which the outgoing eikonal lines meet. Furthermore, these terms have an iterative structure in perturbation theory, in that the extra vertices that contribute can be related to diagrams at a lower order in the perturbation expansion. 
A comment is in order regarding the nature of NE exponentiation. Up to NE order, Eq. (2.20) can be expanded to give

$$
\mathcal{M}=\exp \left[\mathcal{M}^{\mathrm{E}}\right]\left(1+\mathcal{M}_{r}+\mathcal{M}^{\mathrm{NE}}\right)
$$

i.e. one may either consider the $\mathrm{NE}$ terms arising from eikonally factorized diagrams as being in the exponent, or kept to linear order. This masks the fact that the terms in $\mathcal{M}^{\mathrm{NE}}$ genuinely do exponentiate, whereas the remainder terms in $\mathcal{M}_{r}$ do not. However, it is true that the exponentiated NE terms lead to NNE, NNNE etc. contributions which would then mix with higher order (in the eikonal expansion) remainder terms. The exponentiated form of Eq. (4.1) is particularly useful if the contribution from $\mathcal{M}^{\mathrm{NE}}$ (when exponentiated) gives the dominant contribution to higher order terms in the eikonal expansion. Whether or not this is the case is presumably process dependent.

The proof of exponentiation in the abelian case, as presented here, clearly generalizes to higher numbers of eikonal lines. However, in the non-abelian case we considered only the simplest possible hard interaction, namely that with two external lines with a color singlet structure. This could be easily related to a single Wilson line with a cusp. The general case of higher numbers of external lines is more complicated due to the color structures involved. Nevertheless, the methods introduced in this paper may provide a useful starting point in addressing NE corrections in these situations.

We have only considered matrix elements in this paper. Thus, any exponentiation of $\mathrm{NE}$ corrections pertains only before any integration over the phase space of the final state gauge bosons has been performed. In the strict eikonal approximation, the phase space factorizes into a product of single particle phase spaces (i.e. conservation of energy is a subleading effect), thus exponentiation in the matrix element implies exponentiation of soft logarithms in differential (but partially integrated) cross-sections. This is not necessarily the case beyond the eikonal approximation, where one expects NE corrections resulting from the eikonal matrix element with integration over the full phase space. Naïvely, one expects a given differential cross-section (e.g. in some variable $\xi$ related to the total energy fraction carried by soft gluons) to have the form

$$
\frac{d \sigma}{d \xi}=\int d \mathrm{PS}^{(\mathrm{E})}\left|\mathcal{M}^{(\mathrm{E})}\right|^{2}+\left[\int d \mathrm{PS}^{(\mathrm{E})}\left|\mathcal{M}^{(\mathrm{NE})}\right|^{2}+\int d \mathrm{PS}^{(\mathrm{NE})}\left|\mathcal{M}^{(\mathrm{E})}\right|^{2}\right]+\mathcal{O}(\mathrm{NNE}) .
$$

Here $\mathcal{M}^{(\mathrm{E}, \mathrm{NE})}$ denote the eikonal and next-to-eikonal matrix elements respectively, and $d \mathrm{PS}^{(\mathrm{E})}$ the eikonal phase space, consisting of a factorized product of one-particle phase spaces. The first term in Eq.(4.2) is then of eikonal order, and the bracketed term is NE, where $d \mathrm{PS}^{(\mathrm{NE})}$ represents that part of the multi-gluon phase space which implements nextto-eikonal corrections (i.e. subleading terms in $\xi$ ). The precise nature of this latter term is unclear, and an investigation of its effect is deferred to a future publication. Nevertheless, all of the ingredients for the first bracketed term in Eq. (4.2) are contained in this paper.

To conclude, the path integral methods used in this paper provide a new viewpoint for the exponentiation of soft radiative corrections to matrix elements, in both abelian and non-abelian gauge theories. In particular, the discussion of webs is rephrased such that a 
closed form solution for the modified color factors can be given. Furthermore, the approach naturally encompasses the exponentiation of classes of next-to-eikonal corrections. This approach should prove fruitful in the further investigation of soft radiative corrections to all orders in perturbation theory.

\section{Acknowledgments}

We would like to thank Jan-Willem van Holten and Lorenzo Magnea for valuable discussions. The work of EL and CDW is supported by the Netherlands Foundation for Fundamental Research of Matter (FOM) and the National Organization for Scientific Research (NWO).

\section{A. Exponentiation of disconnected diagrams}

Here we briefly prove the exponentiation of disconnected diagrams in quantum field theory, using the replica trick of statistical physics. Although we consider a single self-interacting scalar field $\phi$, the proof generalizes easily to other systems.

The Green's functions of a given quantum field theory are described by the generating functional

$$
Z[J]=\int \mathcal{D} \phi e^{i S[\phi]+i \int J \phi},
$$

where $J$ is a source for the field $\phi$, and $S$ is the classical action. Now consider defining $N$ replicas of the theory, involving fields $\phi_{i}(i \in\{1, \ldots, n\})$. This has generating functional

$$
Z_{N}[J]=\int \mathcal{D} \phi_{1} \ldots \mathcal{D} \phi_{N} e^{i S\left[\phi_{1}\right]+i \int J \phi_{1}} \ldots e^{i S\left[\phi_{N}\right]+i \int J \phi_{N}}
$$

which clearly satisfies

$$
Z_{N}[J]=(Z[J])^{N} .
$$

The Feynman rules for each field are similar, and there are no interactions between the fields. Thus, there can be no more than one field in each connected Feynman diagram, and connected diagrams therefore have $N$ copies. By the same reasoning, disconnected diagrams containing $n \geq 2$ constituent parts have $N^{n}$ copies. It follows that

$$
\sum G_{c} \propto N
$$

where $G_{c}$ denotes a connected diagram. Furthermore, no disconnected diagrams contribute terms proportional to $N$. From Eq. (A.3) one has

$$
Z_{N}[J]=1+N \log (Z[J])+\mathcal{O}\left(N^{2}\right),
$$

and comparing Eqs. (A.4, A.5) gives

$$
\sum G_{c}=\log (Z[J]) .
$$

Finally, one writes this as:

$$
Z[J]=\exp \left[\sum G_{c}\right]
$$


and sets $N=1$. This is the statement that disconnected diagrams exponentiate, as required.

The above proof shortcuts the nontrivial combinatoric fact that the symmetry factor associated with a disconnected Feynman graph is the product of the symmetry factors of each of the constituent connected graphs. The results of this paper demonstrate that the same combinatoric reasoning applies to the exponentiation of soft radiative corrections from fast-moving external particles.

\section{B. Next-to-eikonal Feynman rules}

In this appendix, we show how to derive effective Feynman rules for the eikonal and nextto-eikonal approximations in the path integral approach discussed in section 2.2, and show explicitly that these agree with the rules one obtains by starting from exact perturbation theory before taking the NE limit.

We begin with the conventional perturbative approach, and consider the case of a charged scalar interacting with a background gauge field. The theory and its Green functions are defined via the generating functional

$$
Z\left[J, J^{*}\right]=\int \mathcal{D} \phi \mathcal{D} \phi^{*} \exp \left[i \int d^{d} x\left[-\left(D_{\mu} \phi\right)^{*} D^{\mu} \phi-m^{2} \phi^{*} \phi+J \phi^{*}+J^{*} \phi\right]\right] .
$$

where $D_{\mu}=\partial_{\mu}-i A_{\mu}$. From this functional one derives the Feynman rules

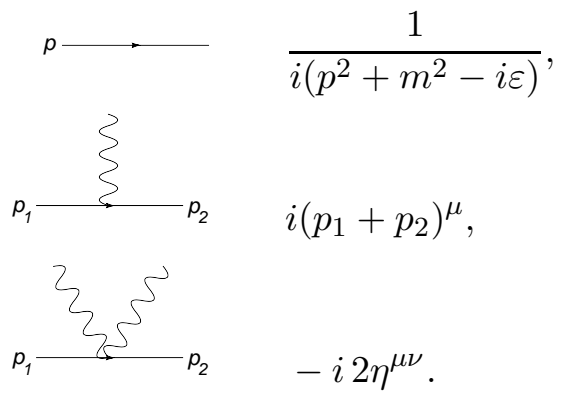

Note the symmetry factor in the seagull vertex. With these rules one can describe emissions from a charged scalar line, and from them we can derive Feynman rules for the eikonal and NE approximation. To do so, we shall take $m=0$ and use $\lambda$-scaling as discussed in section 2. That is, we consider an external particle of 4-momentum $p=\lambda n$, and consider the limit $\lambda \rightarrow \infty$. As discussed in section 2 , this corresponds to the eikonal approximation, with the first subleading corrections in $\lambda$ representing the NE approximation.

We first consider the propagator-vertex combination for one-photon emission. The diagram and corresponding expression is

$$
\rho-k \sum_{-\infty}^{k} \quad \frac{1}{i(p-k)^{2}} i(2 p-k)^{\mu},
$$

where we have taken this diagram to represent the combined vertex and propagator factors (to the left of the vertex). Setting $p=\lambda n$ and expanding Eq. (B.3) to $\mathcal{O}(1 / \lambda)$ yields

$$
-\frac{n^{\mu}}{n \cdot k}+\frac{1}{\lambda}\left(\frac{k^{\mu}}{2 n \cdot k}-k^{2} \frac{n^{\mu}}{2(n \cdot k)^{2}}\right) \text {. }
$$


We recognize the first term as the eikonal approximation, and the remainder as the NE contribution. Each term in Eq. (B.4) is to be contracted with a background gauge field, so that we can treat each as a one-photon source. We represent these sources graphically as

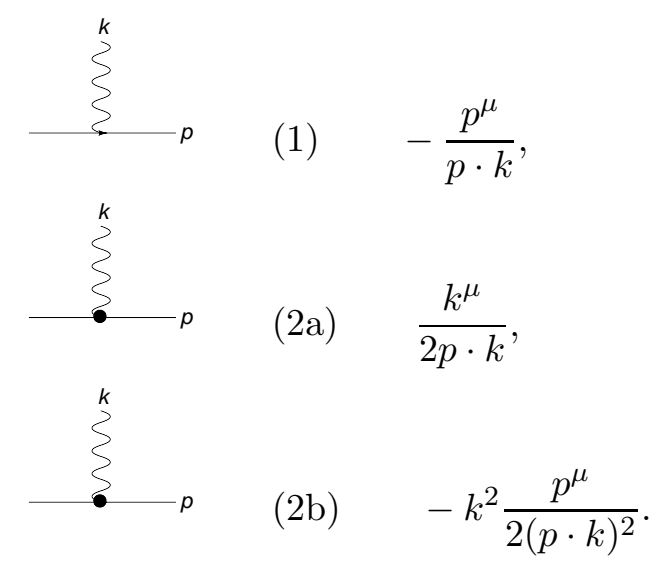

Note that here and in following graphs, we replace $n \rightarrow p / \lambda$ so that the Feynman rules are given in terms of the physical momenta. We now consider possible two-photon sources. Starting with the seagull term, we take the following propagator-vertex combination:

$$
\sum_{p-k-1}^{k} \int^{\prime} \frac{1}{i(p-k-l)^{2}}\left(-i 2 \eta^{\mu \nu}\right)
$$

Scaling $p$ and gathering terms up to $\mathcal{O}(1 / \lambda)$ yields

$$
\frac{1}{\lambda} \frac{\eta^{\mu \nu}}{n \cdot(k+l)}
$$

Evidently, at the eikonal level the seagull term is absent. One expects this given that there is no such seagull vertex in the exact Feynman rules for a fermionic emitting particle, and, as is well known, in the eikonal approximation the emitted radiation is insensitive to the particle's spin.

We next examine the contribution of two individual photon emissions, as shown in Fig. 15. At eikonal level these diagrams give a contribution

$$
\left(-\frac{n^{\nu}}{n \cdot(k+l)}\right)\left(-\frac{n^{\mu}}{n \cdot k}\right)+\left(-\frac{n^{\nu}}{n \cdot(k+l)}\right)\left(-\frac{n^{\mu}}{n \cdot(l)}\right)
$$

which one may rearrange to give:

$$
\left(-\frac{n^{\nu}}{p \cdot k}\right)\left(-\frac{n^{\mu}}{p \cdot l}\right) .
$$

The contribution from the soft emissions explicitly factorizes into a product of uncorrelated emissions, as is well-known.

At $\mathcal{O}(1 / \lambda)$, corresponding to the NE limit, one must sum over all possible insertions of a NE one-photon emission vertex in Fig. (15). There are possible vertices, as given in Eq. (B.5). The vertex (2a) yields an expression 

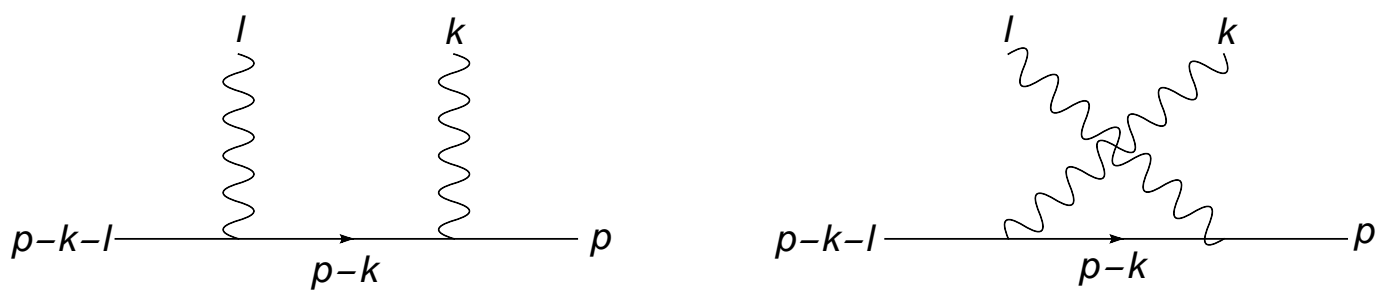

Figure 15: Diagrams contributing to two photon emission. At NE level, one must sum over all possible insertions of a NE one-photon emission vertex, as given in Eq. (B.5).

$$
\begin{aligned}
\left(-\frac{n^{\nu}}{n \cdot(k+l)}\right)\left(\frac{k^{\mu}}{2 n \cdot k}\right)+ & \left(\frac{(2 k+l)^{\nu}}{2 n \cdot(k+l)}\right)\left(-\frac{n^{\mu}}{n \cdot k}\right)+ \\
& \left(-\frac{n^{\mu}}{n \cdot(k+l)}\right)\left(\frac{l^{\nu}}{2 n \cdot l}\right)+\left(\frac{(k+2 l)^{\mu}}{2 n \cdot(k+l)}\right)\left(-\frac{n^{\nu}}{n \cdot l}\right) .
\end{aligned}
$$

which can be rearranged to give

$$
\left(-\frac{n^{\nu}}{n \cdot l}\right)\left(\frac{k^{\mu}}{2 n \cdot k}\right)+\left(-\frac{n^{\mu}}{n \cdot k}\right)\left(\frac{l^{\nu}}{2 n \cdot l}\right)-\frac{l^{\mu} n^{\nu} n \cdot k+k^{\nu} n^{\mu} n \cdot l}{n \cdot(k+l) n \cdot k n \cdot l} .
$$

Notice that the first two terms correspond to two uncorrelated NE emissions, while the last term represents a correlated two-photon emission (i.e. is non-factorizable into terms dependent on a single photon momentum). The NE vertex (2b) gives a contribution

$$
\begin{gathered}
\left(-\frac{n^{\nu}}{n \cdot(k+l)}\right)\left(-k^{2} \frac{n^{\mu}}{2(n \cdot k)^{2}}\right)+\left(-(k+l)^{2} \frac{n^{\nu}}{2(n \cdot(k+l))^{2}}\right)\left(-\frac{n^{\mu}}{n \cdot k}\right)+ \\
\quad\left(-\frac{n^{\mu}}{n \cdot(k+l)}\right)\left(-l^{2} \frac{n^{\nu}}{2(n \cdot l)^{2}}\right)+\left(-(k+l)^{2} \frac{n^{\mu}}{2(n \cdot(k+l))^{2}}\right)\left(-\frac{n^{\nu}}{n \cdot l}\right),
\end{gathered}
$$

which can be rewritten as

$$
\left(-\frac{n^{\nu}}{n \cdot l}\right)\left(-k^{2} \frac{n^{\mu}}{2(n \cdot k)^{2}}\right)+\left(-\frac{n^{\mu}}{n \cdot k}\right)\left(-l^{2} \frac{n^{\nu}}{2(n \cdot l)^{2}}\right)+\frac{n^{\mu} n^{\nu} k \cdot l}{n \cdot(k+l) n \cdot k n \cdot l} .
$$

Again this is the sum of an uncorrelated part, and a term implementing correlated photon emission. The various correlated contributions given in Eqs. (B.7), (B.11) and (B.13) can be represented as new two-photon vertices, and are given respectively by
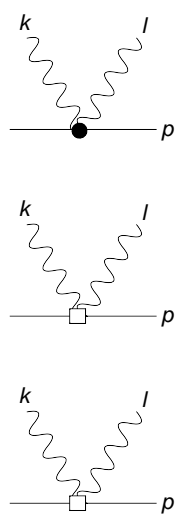$$
+\frac{\eta^{\mu \nu}}{p \cdot(k+l)}
$$$$
-\frac{l^{\mu} p^{\nu} p \cdot k+k^{\nu} p^{\mu} p \cdot l}{p \cdot(k+l) p \cdot k p \cdot l}
$$

$$
+\frac{p^{\mu} p^{\nu} k \cdot l}{p \cdot(k+l) p \cdot k p \cdot l}
$$


We have shown that these vertices apply when two photons are emitted next to the on-shell eikonal line. From our analysis in this appendix, it does not necessarily follow that these vertices apply to emissions anywhere on the external line, at all orders of perturbation theory. That this is indeed the case is clear when one rederives these vertices using the path integral methods described in this paper, and we defer a full proof within conventional perturbation theory to a forthcoming paper [36]. Here we will only demonstrate that these terms are precisely reproduced in our path-integral formalism. The path integral representation of a charged scalar coupled to a background gauge field is given by Eq. (2.16) as

$$
\begin{aligned}
f(\infty)=\int_{x(0)=0} \mathcal{D} x \exp \left[i \int _ { 0 } ^ { \infty } d t \left(\frac{\lambda}{2} \dot{x}^{2}+(n+\dot{x}) \cdot A\left(x_{i}+n t+x\right)\right.\right. & \\
& \left.\left.+\frac{i}{2 \lambda} \partial \cdot A\left(x_{i}+p_{f} t+x\right)\right)\right] .
\end{aligned}
$$

Our task is to derive the photon source terms from this expression. To this end we need to determine the propagator and vertices for the $x$ field, and their scaling with $\lambda$.

The $x$ kinetic term is given by

$$
-\int_{0}^{\infty} d t \frac{1}{2} x(t)\left(i \lambda \frac{\partial^{2}}{\partial t^{2}}\right) x(t),
$$

The propagator for $x$ is given by the inverse of the quadratic operator in Eq. (B.16), which is found to be

$$
G\left(t, t^{\prime}\right)=\frac{i}{\lambda} \min \left(t, t^{\prime}\right) .
$$

Note that it is symmetric, proportional to $1 / \lambda$ (thus is of NE order), and satisfies the condition $G\left(0, t^{\prime}\right)=0$. Other two-point correlators of $x$ and $\dot{x}$, which we need below, are

$$
\begin{gathered}
\left\langle x(t) x\left(t^{\prime}\right)\right\rangle=G\left(t, t^{\prime}\right)=\frac{i}{\lambda} \min \left(t, t^{\prime}\right), \\
\left\langle\dot{x}(t) x\left(t^{\prime}\right)\right\rangle=\frac{\partial G\left(t, t^{\prime}\right)}{\partial t}=\frac{i}{\lambda} \theta\left(t^{\prime}-t\right), \\
\left\langle\dot{x}(t) \dot{x}\left(t^{\prime}\right)\right\rangle=\frac{\partial^{2} G\left(t, t^{\prime}\right)}{\partial t \partial t^{\prime}}=\frac{i}{\lambda} \delta\left(t^{\prime}-t\right) .
\end{gathered}
$$

We will also need the properties of the equal time correlator $\langle x \dot{(t)} x(t)\rangle$. Using the discretization of space-time adopted throughout this paper, the derivative $\dot{x}(t)$ is given by

$$
\lim _{\epsilon \downarrow 0} \epsilon \frac{x(t+\epsilon)-x(t)}{\epsilon}
$$

and thus one has

$$
\langle x \dot{(t)} x(t)\rangle=\frac{i}{\lambda} \lim _{\epsilon \downarrow 0} \frac{\min (t+\epsilon, t)-\min (t, t)}{\epsilon}=0 .
$$

The vertices involving the $x$ field can be obtained by Taylor expansion of the other terms in Eq. (B.15). Due to the subleading nature of the $x$ propagator in the eikonal limit, we 
shall need them to second order in $x$ or $\dot{x}$ only for a NE analysis. The terms without a power of $x$ are

$$
\begin{aligned}
i \int_{0}^{\infty} d t n \cdot A(n t) & =-\int \frac{d^{d} k}{(2 \pi)^{d}} \frac{n^{\mu}}{n \cdot k} \tilde{A}_{\mu}(k), \\
-\frac{1}{2 \lambda} \int_{0}^{\infty} d t \partial \cdot A(n t) & =\frac{1}{2 \lambda} \int \frac{d^{d} k}{(2 \pi)^{d}} \frac{k^{\mu}}{n \cdot k} \tilde{A}_{\mu}(k),
\end{aligned}
$$

where we have also represented the terms in momentum space. Note that these $A$ source terms correspond to the vertex in Eq. (B.5) (2a). Terms with one power of $x$ are

$$
\begin{aligned}
i \int_{0}^{\infty} d t \dot{x}^{\mu} A_{\mu}(n t) & =\int \frac{d^{d} k}{(2 \pi)^{d}} \tilde{A}_{\mu}(k) \int_{0}^{\infty} d t i \dot{x}^{\mu}(t) e^{i(n \cdot k) t} \\
i \int_{0}^{\infty} d t n^{\mu} \partial_{\nu} A_{\mu}(n t) x^{\nu}(t) & =-\int \frac{d^{d} k}{(2 \pi)^{d}} n^{\mu} \tilde{A}_{\mu}(k) k_{\nu} \int_{0}^{\infty} d t x^{\nu}(t) e^{i(n \cdot k) t} \diamond
\end{aligned}
$$

To distinguish the vertices in our discussion below, we have labeled them with symbols. Terms with two powers of $x$ are

$$
\begin{aligned}
i \int_{0}^{\infty} d t \dot{x}^{\mu} \partial_{\nu} A_{\mu}(n t) x^{\nu} & =-\int \frac{d^{d} k}{(2 \pi)^{d}} \tilde{A}_{\mu}(k) k_{\nu} \int_{0}^{\infty} d t \dot{x}^{\mu}(t) x^{\nu}(t) e^{i(n \cdot k) t} \odot \\
\frac{i}{2} \int_{0}^{\infty} d t n^{\mu} \partial_{\nu} \partial_{\kappa} A_{\mu}(n t) x^{\nu}(t) x^{\kappa}(t) & =-\frac{i}{2} \int \frac{d^{d} k}{(2 \pi)^{d}} n^{\mu} \tilde{A}_{\mu}(k) k_{\nu} k_{\kappa} \int_{0}^{\infty} d t x^{\nu}(t) x^{\kappa}(t) e^{i(n \cdot k) t} .
\end{aligned}
$$

The term is quadratic in $x$, and thus the factor of $1 / 2$ does not appear in the resulting vertex.

The next step is to carry out the $x$ path-integral. This amounts to using the Feynman rules in Eqs. (B.17 - B.23), keeping terms to $\mathcal{O}(1 / \lambda)$

$$
\begin{gathered}
\int_{x(0)=0} \mathcal{D} x \exp \left[i \int_{0}^{\infty} d t\left(\frac{\lambda}{2} \dot{x}^{2}+(n+\dot{x}) \cdot A\left(x_{i}+n t+x\right)+\frac{i}{2 \lambda} \partial \cdot A\left(x_{i}+p_{f} t+x\right)\right)\right]= \\
\exp \left[-\int \frac{d^{d} k}{(2 \pi)^{d}} \frac{n^{\mu}}{n \cdot k} \tilde{A}_{\mu}(k)+\frac{1}{2 \lambda} \int \frac{d^{d} k}{(2 \pi)^{d}} \frac{k^{\mu}}{n \cdot k} \tilde{A}_{\mu}(k)+\sum \cdot+\sum \circlearrowright\right] \cdot(\mathrm{B} .24)
\end{gathered}
$$

We have written the result as the exponent of connected diagrams, where terms beyond $\mathcal{O}(1 / \lambda)$ are neglected. The first two terms (B.21) are the vertices (1) and (2a) of (B.5). The third and fourth terms represent tree and loop graphs, with a sum over all possible insertions of the vertices denoted above by $\mathbf{p}, \diamond, \varnothing$ and $\boldsymbol{\phi}$. In the tree graph, there are three different combinations of one- $x$ vertices from (B.22). The $\mathbf{p}$ - $\mathbf{p}$ combination together with a two- $x$ correlator gives

$$
\begin{aligned}
\frac{1}{2} \int \frac{d^{d} k}{(2 \pi)^{d}} \frac{d^{d} k}{(2 \pi)^{d}} \tilde{A}_{\mu}(k) \tilde{A}_{\nu}(l) \int_{0}^{\infty} d t d t^{\prime} \frac{-i}{\lambda} \delta\left(t-t^{\prime}\right) \eta^{\mu \nu} e^{i\left(n \cdot k t+n \cdot l t^{\prime}\right)}= \\
\frac{1}{2} \int \frac{d^{d} k}{(2 \pi)^{d}} \frac{d^{d} k}{(2 \pi)^{d}} \tilde{A}_{\mu}(k) \tilde{A}_{\nu}(l) \frac{\eta^{\mu \nu}}{\lambda n \cdot(k+l)}
\end{aligned}
$$


This precisely reproduces source term (3) of Eq. (B.14). The $-\diamond$ combination gives

$$
\begin{array}{r}
\int \frac{d^{d} k}{(2 \pi)^{d}} \frac{d^{d} k}{(2 \pi)^{d}} \tilde{A}_{\mu}(k) \tilde{A}_{\nu}(l) n^{\nu}\left(-k_{\kappa}\right) \int_{0}^{\infty} d t d t^{\prime} i\left(\frac{i}{\lambda} \delta\left(t-t^{\prime}\right)\right) \eta^{\mu \kappa} \frac{e^{i\left(n \cdot k t+n \cdot l t^{\prime}\right)}}{-i n \cdot l}= \\
\int \frac{d^{d} k}{(2 \pi)^{d}} \frac{d^{d} k}{(2 \pi)^{d}} \tilde{A}_{\mu}(k) \tilde{A}_{\mu}(l) \frac{-n^{\nu} l^{\mu}}{\lambda n \cdot \ln \cdot(k+l)},
\end{array}
$$

which can be rewritten as

$$
\frac{1}{2} \int \frac{d^{d} k}{(2 \pi)^{d}} \frac{d^{d} k}{(2 \pi)^{d}} \tilde{A}_{\mu}(k) \tilde{A}_{\nu}(l)\left(-\frac{n^{\nu} l^{\mu} n \cdot k+n^{\mu} k^{\nu} n \cdot l}{\lambda n \cdot \ln \cdot k n \cdot(k+l)}\right) .
$$

This is source term (4a) of (B.14). The $\diamond-\diamond$ combination gives

$$
\begin{array}{r}
\frac{1}{2} \int \frac{d^{d} k}{(2 \pi)^{d}} \frac{d^{d} k}{(2 \pi)^{d}} \tilde{A}_{\mu}(k) \tilde{A}_{\nu}(l) n^{\mu} n^{\nu} k_{\rho} l_{\sigma} \int_{0}^{\infty} d t d t^{\prime}\left(\frac{i}{\lambda} \delta\left(t-t^{\prime}\right)\right) \eta^{\rho \sigma} \frac{e^{i\left(n \cdot k t+n \cdot l t^{\prime}\right)}}{(-i n \cdot k)(-i n \cdot l)}= \\
\frac{1}{2} \int \frac{d^{d} k}{(2 \pi)^{d}} \frac{d^{d} k}{(2 \pi)^{d}} \tilde{A}_{\mu}(k) \tilde{A}_{\mu}(l) n^{\mu} n^{\nu} \frac{k \cdot l}{\lambda n \cdot \ln \cdot k n \cdot(k+l)} \cdot
\end{array}
$$

which produces term (4b) of (B.14).

The loop graph in Eq. (B.24) has in principle two possible choices of vertex from (B.14). However, the $\varnothing$ vertex does not actually contribute, as it involves the equal time correlator $\langle\dot{x}(t) x(t)\rangle$, which was shown above to be zero. The vertex, however, does contribute and gives

$$
-\frac{1}{2} i \int \frac{d^{d} k}{(2 \pi)^{d}} \frac{d^{d} k}{(2 \pi)^{d}} \tilde{A}_{\mu}(k) k_{\rho} k_{\sigma} \int d t \frac{i}{\lambda} t e^{i n \cdot k t}=-\frac{1}{2} \frac{d^{d} k}{(2 \pi)^{d}} \tilde{A}_{\mu}(k) \frac{k^{2}}{(n \cdot k)^{2}} .
$$

This finally yields source term (2b) of (B.5).

We conclude that, to next-to-eikonal order, the one- and two-photon source terms found by approximations in standard perturbation theory are precisely reproduced in our first-quantized path-integral approach. The considerations of section 2 then show that NE corrections from eikonally factorized diagrams exponentiate to all orders.

We have considered the case of scalar emitting particles in the above discussion. However, things proceed similarly for the spinor case, with the only modification arising due to the presence of an additional term in the exponent of Eq. (B.15)

$$
\frac{i}{2 \lambda} \int_{0}^{\infty} \sigma^{\mu \nu} F_{\mu \nu}=-\frac{1}{\lambda} \int \frac{d^{d} k}{(2 \pi)^{d}} k_{\nu}\left[\gamma^{\nu}, \gamma^{\mu}\right] \tilde{A}_{\mu}(k)
$$

where the right-hand-side corresponds to the momentum space vertex

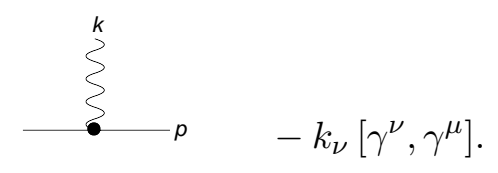

This is $\mathcal{O}(1 / \lambda)$, as expected from the fact that a magnetic moment vertex only contributes for particles having non-zero spin, and radiation in the strictly eikonal limit is insensitive to the spin of the emitting particle.

The above discussion assumes an abelian gauge field. The non-abelian generalization is discussed in Sec. 3 . 


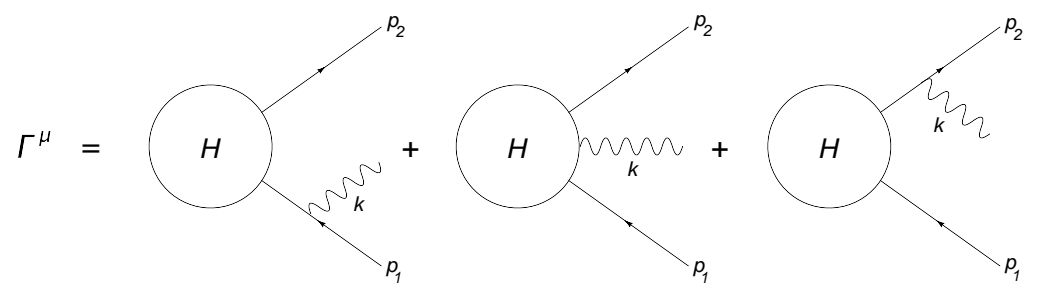

Figure 16: Amplitude $\Gamma^{\mu}$ corresponding to all possible soft emissions from a graph containing a given hard interaction, in the simple case of two outgoing scalar particles. The Lorentz index corresponds to the emitted photon.

\section{Matrix elements with internal emissions}

In section 2.4, we show from our path integral representation of the scattering amplitude how NE corrections arising from soft photon emissions from within the hard interaction can be related to the hard interaction with no emissions, which is part of the content of the Low-Burnett-Kroll theorem [33, 34] (see also [35]). To clarify this discussion, we here present how one would obtain a similar result using traditional Feynman diagram methods, in the case of two scalar lines. Our presentation follows that of e.g. [37].

We consider the momentum-space amplitude $\Gamma^{\mu}$ shown pictorially in Fig. 16, and corresponding to a single gluon emission emitted from a graph containing a given hard interaction with two external scalar particles. We first define $\Gamma \equiv \Gamma\left(p_{1}^{2}, p_{2}^{2}, p_{1} \cdot p_{2}\right)$ to be the hard interaction amplitude with no photon emission. Then using the normal Feynman rules for scalar electrodynamics, one may write $\Gamma^{\mu}$ as

$\Gamma^{\mu}=\frac{\left(2 p_{1}-k\right)^{\mu}}{-2 p_{1} \cdot k} \Gamma\left[\left(p_{1}-k\right)^{2}, p_{2}^{2},\left(p_{1}-k\right) \cdot p_{2}\right]+\frac{\left(2 p_{2}+k\right)^{\mu}}{2 p_{2} \cdot k} \Gamma\left[p_{1}^{2},\left(p_{2}+k\right)^{2}, p_{1} \cdot\left(p_{2}+k\right)\right]+\Gamma_{i n t}^{\mu}$,

where we have assumed light-like external particles, and $\Gamma_{i n t}^{\mu}$ is the amplitude for emission from within the hard interaction, as represented by the second diagram on the right-handside in figure 16. Also, we have neglected coupling constants for brevity. In the limit where $k$ is soft, one may Taylor expand Eq. (C.1) to next-to-leading order in $k$ (corresponding to the NE approximation) to obtain

$$
\begin{aligned}
\Gamma^{\mu} & =\frac{2 p_{1}^{\mu}}{-2 p_{1} \cdot k}\left[\Gamma-2 p_{1} \cdot k \frac{\partial \Gamma}{\partial p_{1}^{2}}-p_{2} \cdot k \frac{\partial \Gamma}{\partial p_{1} \cdot p_{2}}\right]+k^{\mu}\left(\frac{1}{2 p_{1} \cdot k}+\frac{1}{2 p_{2} \cdot k}\right) \\
& +\frac{2 p_{2}^{\mu}}{2 p_{2} \cdot k}\left[\Gamma+2 p_{2} \cdot k \frac{\partial \Gamma}{\partial p_{2}^{2}}+p_{1} \cdot k \frac{\partial \Gamma}{\partial p_{1} \cdot p_{2}}\right]+\Gamma_{i n t}^{\mu} .
\end{aligned}
$$

The gauge invariance condition $k_{\mu} \Gamma^{\mu}=0$ implies

$$
k_{\mu} \Gamma_{i n t}^{\mu}=-2 p_{2} \cdot k \frac{\partial \Gamma}{\partial p_{2}^{2}}-2 p_{1} \cdot k \frac{\partial \Gamma}{\partial p_{1}^{2}}-k \cdot\left(p_{1}+p_{2}\right) \frac{\partial \Gamma}{\partial p_{1} \cdot p_{2}} .
$$

This must be true for arbitrary $k$, so that one may remove factors of $k^{\mu}$ in Eq. (C.3). Then 
one may substitute the resulting form of $\Gamma^{\mu}$ back into Eq. (16) to obtain

$$
\Gamma^{\mu}=\left[\frac{\left(2 p_{1}-k\right)^{\mu}}{-2 p_{1} \cdot k}+\frac{\left(2 p_{2}+k\right)^{\mu}}{2 p_{2} \cdot k}\right] \Gamma+\left[\frac{p_{1}^{\mu}\left(k \cdot p_{2}-k \cdot p_{1}\right)}{p_{1} \cdot k}+\frac{p_{2}^{\mu}\left(k \cdot p_{1}-k \cdot p_{2}\right)}{p_{2} \cdot k}\right] \frac{\partial \Gamma}{\partial p_{1} \cdot p_{2}}
$$

Identifying $p_{i}=n_{i}$ in the notation of section 2.4, one sees that the first term of Eq. (C.4) is the contribution one expects from the effective Feynman rules for soft emission up to NE order. Also, the second term in Eq. (C.4) is precisely the contribution contained in Eq. (2.34). Furthermore, when $p_{1}^{2}=p_{2}^{2}=0$, one may simplify Eq. (C.3) so that one obtains

$$
\Gamma_{i n t}^{\mu}=-p_{1}^{\mu} \frac{\partial \Gamma}{\partial p_{1} \cdot p_{2}}-p_{2}^{\mu} \frac{\partial \Gamma}{\partial p_{1} \cdot p_{2}} .
$$

Rewriting

$$
p_{i}^{\mu} \frac{\partial}{\partial p_{i} \cdot p_{j}}=\frac{\partial}{\partial p_{i}^{\mu}}
$$

one finds

$$
\Gamma_{i n t}^{\mu}=-\sum_{i} \frac{\partial \Gamma}{\partial p_{i}^{\mu}}
$$

which is indeed a special case of Eq. (2.29).

\section{References}

[1] G. Sterman, Summation of Large Corrections to Short Distance Hadronic Cross-Sections, Nucl. Phys. B281 (1987) 310.

[2] S. Catani and L. Trentadue, Resummation of the QCD Perturbative Series for Hard Processes, Nucl. Phys. B327 (1989) 323.

[3] S. Catani and M. Ciafaloni, Generalized coherent state for soft gluon emission, Nucl. Phys. B249 (1985) 301.

[4] G. P. Korchemsky and G. Marchesini, Resummation of large infrared corrections using Wilson loops, Phys. Lett. B313 (1993) 433-440.

[5] G. P. Korchemsky and G. Sterman, Infrared factorization in inclusive B meson decays, Phys. Lett. B340 (1994) 96-108, [http://arXiv.org/abs/hep-ph/9407344].

[6] H. Contopanagos, E. Laenen, and G. Sterman, Sudakov factorization and resummation, Nucl. Phys. B484 (1997) 303-330, [hep-ph/9604313].

[7] S. Forte and G. Ridolfi, Renormalization group approach to soft gluon resummation, Nucl. Phys. B650 (2003) 229-270, [hep-ph/0209154].

[8] T. Becher and M. Neubert, Threshold resummation in momentum space from effective field theory, Phys. Rev. Lett. 97 (2006) 082001, [hep-ph/0605050].

[9] D. R. Yennie, S. C. Frautschi, and H. Suura, The infrared divergence phenomena and high-energy processes, Ann. Phys. 13 (1961) 379-452.

[10] J. G. M. Gatheral, Exponentiation of eikonal cross-sections in nonabelian gauge theories, Phys. Lett. B133 (1983) 90. 
[11] J. Frenkel and J. C. Taylor, Nonabelian eikonal exponentiation, Nucl. Phys. B246 (1984) 231.

[12] G. Sterman, Infrared divergences in perturbative QCD, . in Tallahassee 1981, Proceedings, Perturbative Quantum Chromodynamics, 22-40.

[13] C. F. Berger, Soft gluon exponentiation and resummation, hep-ph/0305076. PhD Thesis.

[14] M. J. Strassler, Field theory without Feynman diagrams: One loop effective actions, Nucl. Phys. B385 (1992) 145-184, [hep-ph/9205205].

[15] M. G. Schmidt and C. Schubert, Worldline Green functions for multiloop diagrams, Phys. Lett. B331 (1994) 69-76, [hep-th/9403158].

[16] J. W. van Holten, Propagators and path integrals, Nucl. Phys. B457 (1995) 375-407, [hep-th/9508136].

[17] Z. Bern and D. C. Dunbar, A Mapping between Feynman and string motivated one loop rules in gauge theories, Nucl. Phys. B379 (1992) 562-601.

[18] Z. Bern and D. A. Kosower, The Computation of loop amplitudes in gauge theories, Nucl. Phys. B379 (1992) 451-561.

[19] A. I. Karanikas, C. N. Ktorides, and N. G. Stefanis, World-line techniques for resumming gluon radiative corrections at the cross-section level, Eur. Phys. J. C26 (2003) 445-455, [hep-ph/0210042].

[20] M. Mezard, G. Parisi, and M. Virasoro, Spin Glass Theory and Beyond, . World Scientific (1987) 476pp.

[21] M. Kramer, E. Laenen, and M. Spira, Soft gluon radiation in Higgs boson production at the LHC, Nucl. Phys. B511 (1998) 523, [hep-ph/9611272].

[22] S. Catani, D. de Florian, and M. Grazzini, Higgs production in hadron collisions: Soft and virtual QCD corrections at NNLO, JHEP 0105 (2001) 025, [http://arXiv.org/abs/hep-ph/0102227].

[23] R. V. Harlander and W. B. Kilgore, Soft and virtual corrections to $p p \rightarrow H+X$ at $N N L O$, Phys. Rev. D64 (2001) 013015, [hep-ph/0102241].

[24] S. Catani, D. de Florian, M. Grazzini, and P. Nason, Soft-gluon resummation for Higgs boson production at hadron colliders, JHEP 0307 (2003) 028, [hep-ph/0306211].

[25] N. Kidonakis, Collinear and soft gluon corrections to Higgs production at NNNLO, Phys. Rev. D77 (2008) 053008, [0711.0142].

[26] R. Basu, E. Laenen, A. Misra, and P. Motylinski, Soft-collinear effects in prompt photon production, Phys. Rev. D76 (2007) 014010, [0704.3180].

[27] E. Laenen, L. Magnea, and G. Stavenga, On next-to-eikonal corrections to threshold resummation for the Drell-Yan and DIS cross sections, Phys. Lett. B669 (2008) 173-179, [0807.4412].

[28] Y. L. Dokshitzer, G. Marchesini, and G. P. Salam, Revisiting parton evolution and the large- $x$ limit, Phys. Lett. B634 (2006) 504-507, [hep-ph/0511302].

[29] L. Brink, P. Di Vecchia, and P. S. Howe, A Locally Supersymmetric and Reparametrization Invariant Action for the Spinning String, Phys. Lett. B65 (1976) 471-474. 
[30] A. G. Morgan, Second order fermions in gauge theories, Phys. Lett. B351 (1995) 249-256, [hep-ph/9502230].

[31] C. S. Lam, Spinor helicity technique and string reorganization for multiloop diagrams, Can. J. Phys. 72 (1994) 415-438, [hep-ph/9308289].

[32] G. Sterman, Summation of large corrections to short distance hadronic cross-sections, Nucl. Phys. B281 (1987) 310.

[33] F. E. Low, Bremsstrahlung of very low-energy quanta in elementary particle collisions, Phys. Rev. 110 (1958) 974-977.

[34] T. H. Burnett and N. M. Kroll, Extension of the low soft photon theorem, Phys. Rev. Lett. 20 (1968) 86 .

[35] V. Del Duca, High-energy bremsstrahlung theorems for soft photons, Nucl. Phys. B345 (1990) 369-388.

[36] E. Laenen, L. Magnea, G. Stavenga, and C. D. White. In preparation.

[37] G. Sterman, An Introduction to Quantum Field Theory, . Cambridge, UK: Univ. Pr. (1993) 572 pp. 